\title{
Separability of double cosets and conjugacy classes in 3-manifold groups
}

\author{
Emily Hamilton, Henry Wilton and Pavel Zalesskii
}

November 5, 2018

\begin{abstract}
Let $M=\mathbb{H}^{3} / \Gamma$ be a hyperbolic 3-manifold of finite volume. We show that if $H$ and $K$ are abelian subgroups of $\Gamma$ and $g \in \Gamma$, then the double coset $H g K$ is separable in $\Gamma$. As a consequence we prove that if $\mathrm{M}$ is a closed, orientable, Haken 3-manifold and the fundamental group of every hyperbolic piece of the torus decomposition of $M$ is conjugacy separable then so is the fundamental group of $M$. Invoking recent work of Agol and Wise, it follows that if $M$ is a compact, orientable 3-manifold then $\pi_{1}(M)$ is conjugacy separable.
\end{abstract}

\section{Introduction}

The profinite topology on a group $\Gamma$ is the coarsest topology in which every homomorphism from $\Gamma$ to a finite group is continuous. When $\Gamma$ is the fundamental group of a manifold $M$, the profinite topology on $\Gamma$ encodes the finitesheeted covering spaces of $M$, and as such is of great interest in the field of low-dimensional topology.

Definition 1.1. If a subset $X$ of $\Gamma$ is closed in the profinite topology then $X$ is called separable. Equivalently, for every $\gamma \in \Gamma-X$ there is a homomorphism $\phi$ from $\Gamma$ to a finite group such that $\phi(\gamma) \notin \phi(X)$.

1. A group $\Gamma$ is residually finite if the trivial subgroup is separable in $\Gamma$.

2. A group $\Gamma$ is conjugacy separable if every conjugacy class in $\Gamma$ is separable.

3. A group $\Gamma$ is subgroup separable or locally extended residually finite (LERF) if every finitely generated subgroup of $\Gamma$ is separable in $\Gamma$.

4. A group $\Gamma$ is double-coset separable if for every pair $H, K$ of finitely generated subgroups of $\Gamma$, and every $g \in \Gamma$ the double coset $H g K$ is separable.

Note that conjugacy separability and subgroup separability both imply residual finiteness, while double-coset separability implies subgroup separability. 
Hempel showed, using Thurston's Geometrization Theorem, that the fundamental groups of Haken 3-manifolds are residually finite [17. Scott proved that the fundamental groups of surfaces and Seifert-fibered 3-manifolds are subgroup separable [29], and asked whether the same holds for all 3-manifold groups. Burns, Karass and Solitar 9] answered this question in the negative by giving an example of a graph manifold with non-subgroup-separable fundamental group, but it follows from the main theorems of recent preprints of Agol [2] and Wise 34 that the fundamental groups of hyperbolic 3-manifolds are subgroup separable. In this paper, we are concerned with conjugacy separability and double-coset separability in 3-manifold groups.

\subsection{Conjugacy separability}

Our first theorem extends the results of [32], in which it was proved that the fundamental groups of graph manifolds are conjugacy separable.

Theorem 1.2. Let $M$ be a closed, orientable, Haken 3-manifold and let $N_{1}, \ldots, N_{m}$ be the pieces of the torus decomposition of $M$. If each $\pi_{1}\left(N_{i}\right)$ is conjugacy separable then $\pi_{1}(M)$ is conjugacy separable.

This is an important step in the proof that the fundamental group of any compact, orientable 3-manifold is conjugacy separable, as we now explain. We follow the same broad strategy that Hempel used in his proof of residual finiteness, although there are more difficult technical obstacles to overcome.

Let $M$ be a compact, orientable 3-manifold, possibly with boundary. We are interested in the question of whether or not $\pi_{1}(M)$ is conjugacy separable. If $M$ has boundary then, cutting along compressing discs and appealing to the fact that a free product of conjugacy separable groups is conjugacy separable 31, we may assume that $\partial M$ is incompressible. Let $D$ be the double of $M$ along $\partial M$. Then $\pi_{1}(M)$ is a retract of $\pi_{1}(D)$. In particular, a pair of elements is conjugate in $\pi_{1}(M)$ if and only if it is conjugate in $\pi_{1}(D)$, and it follows that if $\pi_{1}(D)$ is conjugacy separable then so is $\pi_{1}(M)$. In this way, we can reduce to the case in which $M$ is orientable and closed. Passing to the pieces of the Kneser-Milnor decomposition, and appealing again to the fact that a free product of conjugacy separable groups is conjugacy separable, we can reduce further to the irreducible case.

The next step is to pass to the pieces of the torus decomposition of $M$, described by Jaco-Shalen [18 and Johannson 20. This point is the heart of Hempel's argument. He proves a gluing theorem that reduces the residual finiteness of $\pi_{1}(M)$ to the residual finiteness of the fundamental groups of the pieces. In the context of conjugacy separability, Theorem 1.2 supplies the necessary gluing theorem.

This reduces the question of which 3-manifold groups are conjugacy separable to the geometric case. If $M$ is a torus bundle over a circle then $\pi_{1}(M)$ is polycyclic, and so is conjugacy separable by a theorem of Remeslennikov [28]. Martino proved that the fundamental groups of Seifert-fibered 3-manifolds are conjugacy separable [24]. 
The hyperbolic case is much more difficult, but dramatic progress has been made recently. The crucial concept is the notion of a special group, introduced by Haglund and Wise [13]1. Minasyan proved that special groups are conjugacy separable [26, while Chagas and the third author gave conditions under which conjugacy separability passes to finite extensions [10. Taking these two results together, it follows that, if $N$ is a hyperbolic 3-manifold and $\pi_{1}(N)$ is virtually special (that is, $\pi_{1}(N)$ has a special subgroup of finite index) then $\pi_{1}(N)$ is conjugacy separable.

Many hyperbolic 3-manifold and orbifold groups are known to be virtually special 17, 8, 10, 11, among them non-cocompact arithmetic and standard cocompact arithmetic lattices. Wise has announced a proof that the fundamental group of any hyperbolic 3-manifold containing an embedded geometrically finite surface is virtually special [33; the heart of his proof is contained in 34. Very recently, Agol has given a proof that the fundamental group of any closed hyperbolic 3-manifold group is virtually special [2. His proof uses the work of 34], as well as Kahn and Markovic's resolution of the Surface Subgroup Conjecture [21] and an extension of the techniques of [1].

Combining the recent work of Wise and Agol with Theorem 1.2 we deduce that the fundamental group of every closed, orientable 3-manifold is conjugacy separable. As described above, conjugacy separability for any compact, orientable 3-manifold, possibly with boundary, reduces to the closed case. We therefore have a complete resolution of the question of conjugacy separability for the fundamental groups of compact, orientable 3-manifolds.

Theorem 1.3. If $M$ is any compact, orientable 3-manifold then $\pi_{1}(M)$ is conjugacy separable.

We emphasize again that Theorem 1.3 depends on the results of [2] and 34].

\subsection{Double-coset separability}

In [32, the second and third authors proved a combination theorem for conjugacyseparable groups, and were able to check the hypotheses in the case when the vertex groups are the fundamental groups of Seifert-fibered 3-manifolds. To prove Theorem 1.2, we need to check the hypotheses of the combination theorem for the fundamental groups of hyperbolic 3-manifolds of finite volume. In particular, we need to prove that double cosets of peripheral subgroups of Kleinian groups of finite covolume are separable. In fact, we prove the following more general result.

Theorem 1.4 (Theorem 3.2). Let $M=\mathbb{H}^{3} / \Gamma$ be a hyperbolic 3-orbifold of finite volume. If $H$ and $K$ are abelian subgroups of $\Gamma$ and $g \in \Gamma$, then the double coset $H g K=\{h g k \mid h \in H, k \in K\}$ is separable in $\Gamma$.

In the closed case, this result can also be deduced from the subgroup separability of $\Gamma$ [2]. Indeed, Minasyan proved that, if $G$ is a word-hyperbolic group

\footnotetext{
${ }^{1}$ By a special group we shall mean a group that is the fundamental group of a compact A-special cube complex. The reader is referred to [13] for definitions.
} 
(as $\Gamma$ is when $M$ is closed), and every quasi-convex subgroup $H$ is separable in $G$, then for any pair of quasi-convex subgroups $H$ and $K$ and any $g \in G$ the double coset $H g K$ is separable [25]. (Abelian subgroups of a word-hyperbolic group are always quasi-convex.) However, Minasyan's theorem has not been generalized to the case in which $M$ has boundary.

Haglund and Wise prove that double cosets of hyperplane subgroups are separable in virtually special groups - see [13, to which the reader is also referred for the definition of a hyperplane subgroup. When $\Gamma$ is virtually special and $H$ is a hyperplane subgroup of $\Gamma$ then $\Gamma$ has a finite-index subgroup that splits as an amalgamated product or HNN extension over $H$. It follows that if $\Gamma$ is a Kleinian group of finite covolume then no hyperplane subgroup of $\Gamma$ is abelian.

\subsection{An outline}

This paper is structured as follows. In Section 2 we state the algebraic results underlying the proof of Theorem 1.4. In Section 3 we prove Theorem 1.4 and

discuss some implications. In Section 4 we recall the results of 32 and prove Theorem 1.2 .

\section{Acknowledgments}

The first author would like to thank Eric Brussel and Alan Reid for useful conversations. The second author would like to thank Stefan Friedl for some comments on an earlier draft; he is supported by an EPSRC Career Acceleration Fellowship. The third author is supported by CNPq.

\section{Algebraic preliminaries}

In this section we prove algebraic results that will be used in the proof of Theorem 1.4. We assume standard terminology of algebraic number theory. For reference see [19].

Notation 2.1. By a number field we mean a finite field extension of $\mathbb{Q}$. If $k$ is a number field, let $\mathcal{O}_{\mathrm{k}}$ denote the ring of algebraic integers of $k$. If $\mathfrak{p}$ is a non-zero prime ideal of $\mathcal{O}_{\mathrm{k}}$, then we complete $k$ at $\mathfrak{p}$ to obtain the local field $k_{\mathfrak{p}}$, with ring of algebraic integers $\mathcal{O}_{\mathrm{k}_{\mathfrak{p}}}$. The ring $\mathcal{O}_{\mathrm{k}_{\mathfrak{p}}}$ has a unique maximal ideal. The quotient of $\mathcal{O}_{\mathrm{k}_{\mathfrak{p}}}$ by this maximal ideal is called the residue class field of $\mathcal{O}_{\mathrm{k}_{\mathfrak{p}}}$. The quotient map is called the residue class field map with respect to $\mathfrak{p}$.

We begin by stating two theorems and two corollaries from [16].

Theorem 2.2. Let $k$ be a number field and let $\delta$ be a non-zero element of $k$ that is not a root of unity. Let $S$ be a finite set of prime ideals of $\mathcal{O}_{\mathrm{k}}$. Then there exists a positive integer $n$ with the following property. For each integer $m \geq n$, there exists a non-zero prime ideal $\mathfrak{p}$ of $\mathcal{O}_{\mathrm{k}}$, lying outside of $S$, such that $\delta \in \mathcal{O}_{\mathrm{k}_{\mathfrak{p}}}$ and the multiplicative order of the image of $\delta$ in the residue class field of $\mathcal{O}_{\mathrm{k}_{\mathfrak{p}}}$ is equal to $\mathrm{m}$. 
For the proof of Theorem 2.2 please see Theorem 2.3 of [16].

Corollary 2.3. Let $R$ be a finitely generated ring in a number field $k$, let $\delta$ be a non-zero element of $R$ that is not a root of unity, and let $x_{1}, x_{2}, \ldots, x_{j}$ be non-zero elements of $R$. Then there exists a positive integer $n$ with the following property. For each integer $m \geq n$, there exist a finite field $F$ and a ring homomorphism $\eta: R \rightarrow F$ such that the multiplicative order of $\eta(\delta)$ is equal to $m$ and $\eta\left(x_{i}\right) \neq 0$, for each $1 \leq i \leq j$.

The deduction of Corollary 2.3 from Theorem 2.2 is given in [16]. (See Corollary 2.5 of [16].) We include the proof in this paper for the convenience of the reader.

Proof. Fix a finite generating set $G$ of $R$. Let $S$ denote the finite set of prime ideals of $\mathcal{O}_{\mathrm{k}}$ which divide an element of $\left\{G, x_{1}, x_{2}, \ldots, x_{j}\right\}$. By Theorem 2.2 . there exists a positive integer $n$ with the following property. For each integer $m \geq n$ there exists a non-zero prime ideal $\mathfrak{p}$ of $\mathcal{O}_{\mathrm{k}}$, lying outside of $S$, such that $\delta \in \mathcal{O}_{\mathrm{k}_{\mathrm{p}}}$ and the multiplicative order of the image of $\delta$ in the residue class field of $\mathcal{O}_{\mathrm{k}_{\mathrm{p}}}$ is equal to $m$. Fix $m \geq n$ and let $\mathfrak{p} \subset \mathcal{O}_{\mathrm{k}}$ be the corresponding prime ideal. Let $F$ denote the residue class field of $\mathcal{O}_{\mathrm{k}_{\mathfrak{p}}}$ and let $\eta: \mathcal{O}_{\mathrm{k}_{\mathfrak{p}}} \rightarrow F$ denote the residue class field map with respect to $\mathfrak{p}$. Since $\mathfrak{p} \notin S, R \in \mathcal{O}_{\mathrm{k}_{\mathfrak{p}}}$ and $x_{1}, x_{2}, \ldots, x_{j}$ are units in $\mathcal{O}_{\mathrm{k}_{\mathrm{p}}}$. Therefore, the restriction of $\eta$ to $R$ satisfies the conclusion of the corollary.

Theorem 2.4. Let $k$ be a number field. Let $\lambda$ and $\omega$ be non-zero elements of $k$ such that $\lambda$ is not a multiplicative power of $\omega$. Let $P$ be a finite set of prime ideals of $\mathcal{O}_{\mathrm{k}}$. Then there exist primes $\mathfrak{p}$ and $\mathfrak{q}$, lying outside of $P$, such that $\lambda, \omega \in \mathcal{O}_{\mathrm{k}_{\mathfrak{p}}} \cap \mathcal{O}_{\mathrm{k}_{\mathfrak{q}}}$ and $\left(\eta_{\mathfrak{p}} \times \eta_{\mathfrak{q}}\right)(\lambda)$ is not a multiplicative power of $\left(\eta_{\mathfrak{p}} \times \eta_{\mathfrak{q}}\right)(\omega)$.

For the proof of Theorem 2.4 please see Theorem 2.7 of [16].

Corollary 2.5. Let $R$ be a finitely generated ring in a number field $k$. Let $\lambda$ and $\omega$ be non-zero elements of $R$ such that $\lambda$ is not a multiplicative power of $\omega$. Then there exist a finite ring $S$ and a ring homomorphism $\eta: R \rightarrow S$ such that $\eta(\lambda)$ is not a multiplicative power of $\eta(\omega)$.

The deduction of Corollary 2.5 from Theorem 2.4 is similar to the deduction of Corollary 2.3 from Theorem 2.2. See Corollary 2.8 of [16] for details.

Theorem 2.4 can be interpreted as a multiplicative subgroup separability result for finitely generated rings lying in number fields. We conclude this section with an additive subgroup separability result.

Theorem 2.6. Let $R$ be a finitely generated ring in a number field $k$. By fixing $a \mathbb{Q}$ embedding of $k$ into $\mathbb{C}$, we may view $k \subset \mathbb{C}$. Let $\beta$ be an element of $R$ and set $A=\{m+n \beta \mid m, n \in \mathbb{Z}\}$ and $B=\{m+n \beta \mid m, n \in \mathbb{Q}\}$. If $b \in R-B$, then there exist a finite ring $S$ and a ring homomorphism $\eta: R \rightarrow S$ such that $\eta(b) \notin \eta(A)$. 
Proof. We first consider the case where $b \notin \mathbb{Q}(\beta)$. Let $L$ denote the normal closure of $k$ over $\mathbb{Q}$. Since $b \notin \mathbb{Q}(\beta)$, there exists an element $\sigma \in \operatorname{Gal}(L / \mathbb{Q})$ such that $\sigma(b) \neq b$ and $\sigma$ fixes $\mathbb{Q}(\beta)$ pointwise. By the Tchebotarev Density Theorem, there are infinitely many primes $p$ of $\mathbb{Q}$ with unramified extension $\mathfrak{p}$ in $L$ such that $\sigma$ is the Frobenius automorphism for $\mathfrak{p} / p$. Fix one such $\mathfrak{p} / p$ such that $R \subset \mathcal{O}_{L_{\mathfrak{p}}}$, where $\mathcal{O}_{L_{\mathfrak{p}}}$ denotes the ring of integers in the $\mathfrak{p}$-adic field $L_{\mathfrak{p}}$. Such a choice is possible since $R$ is finitely generated, and each generator is an integer in $L_{\mathfrak{p}}$ for all but finitely many $\mathfrak{p}$. Let $F_{\mathfrak{p}}$ denote the residue class field of $\mathcal{O}_{L_{p}}$ and let $\mathbb{F}_{p}$ denote the finite field of $p$ elements. Let $\eta$ be the composition of the inclusion map of $R$ into $\mathcal{O}_{\mathrm{L}_{\mathfrak{p}}}$ with the residue map:

$$
\eta: R \hookrightarrow \mathcal{O}_{\mathrm{L}_{\mathfrak{p}}} \rightarrow F_{\mathfrak{p}} .
$$

Since $\sigma$ is the Frobenius automorphism of $L / \mathbb{Q}$ with respect to $\mathfrak{p} / p, \operatorname{Gal}\left(L_{\mathfrak{p}} / \mathbb{Q}_{p}\right)=$ $\left\langle\sigma^{\prime}\right\rangle$ where $\sigma^{\prime}=\sigma$ on $L$. Since $A \subset \mathbb{Q}(\beta)$ and $\sigma$ fixes $\mathbb{Q}(\beta)$ pointwise, $A \subset \mathbb{Q}_{p}$. Since $\sigma(b) \neq b, b \notin \mathbb{Q}_{p}$. The Galois group of $F_{\mathfrak{p}} / \mathbb{F}_{p}$ is also induced by $\sigma$. It follows that $\eta(A) \subset \mathbb{F}_{p}$, but $\eta(b) \notin \mathbb{F}_{p}$. Therefore, $\eta$ and $S=\mathbb{F}_{p}$ satisfy the conclusion of the theorem.

Now consider the case where $b \in \mathbb{Q}(\beta)$. Let $f$ be the minimal monic polynomial of $\beta$ over $\mathbb{Q}$ and let $n$ be the degree of $f$. Our assumption that $b \notin B$ implies that $n>2$. Moreover, we can express $b=a_{0}+a_{1} \beta+a_{2} \beta^{2}+\ldots+a_{n-1} \beta^{n-1}$, where $a_{i} \in \mathbb{Q}$ and at least one coefficient $a_{i_{0}} \in\left\{a_{2}, a_{3}, \ldots, a_{n-1}\right\}$ is non-zero. Since $R$ is a finitely generated ring consisting of algebraic numbers, $R$ is integral over $\mathbb{Z}[1 / s]$, for all but finitely many integers $s$. Choose $s$ such that the coefficients of $f$ are in $\mathbb{Z}[1 / s]$ and $a_{i} \in \mathbb{Z}[1 / s], \forall i \in\{0,1,2, \ldots, n-1\}$. Let $\mathbb{Z}[1 / s][T]$ denote the polynomial ring of $\mathbb{Z}[1 / s]$. Let $p$ be a prime that does not divide $s$ or the numerator of $a_{i_{0}}$ and let $\mathbb{F}_{p}$ denote the finite field of $p$ elements. Since $f$ is a monic polynomial, the map

$$
\mathbb{Z}[1 / s][T] \rightarrow \mathbb{Z}[1 / s][\beta] \text {, given by } T \rightarrow \beta,
$$

is an epimorphism with kernel $(f)$. Therefore, the map

$$
\rho: \mathbb{Z}[1 / s][\beta] \rightarrow \mathbb{Z}[1 / s][T] /(f), \text { given by } \beta \rightarrow T+(f),
$$

is an isomorphism. The quotient map

$$
\mathbb{Z}[1 / s] \rightarrow \mathbb{Z}[1 / s] /(p) \cong \mathbb{F}_{p}
$$

induces

$$
\phi: \mathbb{Z}[1 / s][T] /(f) \rightarrow \mathbb{F}_{p}[T] /(\bar{f}),
$$

where $\bar{f}$ is the image of $f$ in the polynomial ring $\mathbb{F}_{p}[T]$. Write $\bar{f}=\bar{f}_{1} \bar{f}_{2} \ldots \bar{f}_{m}$ as a product of irreducible factors in $\mathbb{F}_{p}[T]$, and let $F_{i}=\mathbb{F}_{p}[T] /\left(\bar{f}_{i}\right)$. Then the natural map

$$
\psi: \mathbb{F}_{p}[T] /(\bar{f}) \rightarrow F_{1} \times F_{1} \times \ldots \times F_{m}
$$

is an isomorphism. Let

$$
\rho_{i}: \mathbb{Z}[1 / s][\beta] \rightarrow \mathbb{Z}[1 / s][T] /(f) \rightarrow \mathbb{F}_{p}[T] /(\bar{f}) \rightarrow F_{1} \times F_{1} \times \ldots \times F_{m} \rightarrow F_{i}
$$


be the composition of $(\psi \phi \rho)$ with the projection of $F_{1} \times F_{1} \times \ldots \times F_{m}$ onto $F_{i}$. Since $F_{i}$ is a field, the kernel of $\rho_{i}$ is a maximal ideal $\mathfrak{p}_{i}$ of $\mathbb{Z}[1 / s][\beta]$. By replacing $R$ with $R[1 / s]$, if necessary, we may assume that $1 / s \in R$. Thus, $R$ is an integral extension of $\mathbb{Z}[1 / s][\beta]$. Therefore, there exists a maximal ideal $\mathfrak{q}_{i}$ of $R$ such that $(\mathbb{Z}[1 / s][\beta]) \cap \mathfrak{q}_{i}=\mathfrak{p}_{i}$. Let $\eta_{i}$ denote the quotient map $R \rightarrow R / \mathfrak{q}_{i}$. Note that the restriction of $\eta_{i}$ to $\mathbb{Z}[1 / s][\beta]$ is equal to $\rho_{i}$. Let $\pi_{i}: R / \mathfrak{q}_{1} \times$ $R / \mathfrak{q}_{2} \times \ldots \times R / \mathfrak{q}_{m} \rightarrow R / \mathfrak{q}_{i}$ denote the projection onto the $i$-th factor. By the universal property of direct products of rings, there exists a unique ring homomorphism $\eta: R \rightarrow R / \mathfrak{q}_{1} \times R / \mathfrak{q}_{2} \times \ldots \times R / \mathfrak{q}_{m}$ such that $\pi_{i} \circ \eta=\eta_{i}$. We claim that $\eta(b) \notin \eta(A)$. Assume, to the contrary, that $\eta(b)=\eta\left(c_{0}+c_{1} \beta\right)$ for some $c_{0}+c_{1} \beta \in A$. Then

$$
b-\left(c_{0}+c_{1} \beta\right)=\left(a_{0}-c_{0}\right)+\left(a_{1}-c_{1}\right) \beta+a_{2} \beta^{2}+\ldots+a_{n-1} \beta^{n-1}
$$

is in the kernel of $\rho_{i}$ for every $i \in\{1,2, \ldots, m\}$. Let

$$
h(T)=\left(a_{0}-c_{0}\right)+\left(a_{1}-c_{1}\right) T+a_{2} T^{2}+\ldots+a_{n-1} T^{n-1} \in \mathbb{Z}[1 / s][T]
$$

and let $\bar{h}$ be the image of $h$ in $\mathbb{F}_{p}[T]$. Then $\bar{f}$ divides $\bar{h}$ in $\mathbb{F}_{p}[T]$. But this contradicts the fact that the image of $a_{i_{0}}$ is non-zero in $\mathbb{F}_{p}$ and so $1<\operatorname{deg}(\bar{h})<$ $n$. Therefore, $\eta$ and $S=R / \mathfrak{q}_{1} \times R / \mathfrak{q}_{2} \times \ldots \times R / \mathfrak{q}_{m}$ satisfy the conclusion of the theorem.

\section{Proof of double-coset separability}

In this section we prove that double cosets of abelian subgroups of Kleinian groups of finite covolume are separable. The proof will use the following proposition from 27.

Proposition 3.1. Let $G_{0}, H$ and $K$ be finitely generated subgroups of a group $G$ and set $H_{0}=H \cap G_{0}$ and $K_{0}=K \cap G_{0}$. If $\left[G: G_{0}\right]$ is finite and if $H_{0} K_{0}$ is separable in $G_{0}$, then $H K$ is separable in $G$.

For a proof of this result see Proposition 2.2 of $[27$.

Theorem 3.2. Let $M=\mathbb{H}^{3} / \Gamma$ be an orientable hyperbolic 3-orbifold of finite volume. Let $H$ and $K$ be abelian subgroups of $\Gamma$, and let $g \in \Gamma$. Then the double coset $H g K=\{h g k \mid h \in H, k \in K\}$ is separable in $\Gamma$.

Proof. As noted in 27, since the profinite topology on $\Gamma$ is equivariant under left and right multiplication, the double coset $\mathrm{HgK}$ is closed in $\Gamma$ if and only if $H^{g} K=g^{-1} H g K$ is closed in $\Gamma$. Therefore, to prove the theorem, it suffices to show that if $H$ and $K$ are abelian subgroups of $\Gamma$, then the double coset $H K$ is separable in $\Gamma$. Note that $g \in H K$ if and only if $g^{-1} \in K H$. Therefore, $H K$ is separable in $\Gamma$ if and only if $K H$ is separable in $\Gamma$.

By Selberg's Lemma [4, $\Gamma$ has a subgroup of finite index $\Gamma_{0}$ which is torsion free. Let $H_{0}=H \cap \Gamma_{0}$ and $K_{0}=K \cap \Gamma_{0}$. By Proposition 3.1. if $H_{0} K_{0}$ is 
separable in $\Gamma_{0}$, then $H K$ is separable in $\Gamma$. Therefore, we may assume that $\Gamma$ is torsion free, and thus the fundamental group of a hyperbolic manifold. Since abelian subgroups of finitely generated Kleinian groups are separable [3], we may assume that $H$ and $K$ do not commute. In particular, both $H$ and $K$ are non-trivial. Since $\Gamma$ is torsion free, the non-trivial abelian subgroups of $\Gamma$ are free abelian of rank 1 or 2 . The free abelian subgroups of rank 1 can be generated by loxodromic or parabolic isometries. The free abelian subgroups of rank 2 are generated by parabolic isometries. If $H$ has rank 1 , let $H^{\prime}$ be the maximal cyclic subgroup of $\Gamma$ containing $H$. If $H$ has rank 2 , let $H^{\prime}$ be the maximal abelian subgroup of $\Gamma$ containing $H$. Then $H$ has finite index in $H^{\prime}$. Let $\left\{a_{1}, a_{2}, \ldots, a_{n-1}\right\}$ be a set of non-trivial coset representatives of $H^{\prime} / H$. By [3, $H$ is a separable subgroup of $\Gamma$. Therefore, there exists a subgroup $\Gamma_{H}$ of finite index in $\Gamma$ such that $H \subset \Gamma_{H}$ but $\Gamma_{H} \cap\left\{a_{1}, a_{2}, \ldots, a_{n-1}\right\}=\emptyset$. Then $\Gamma_{H} \cap H^{\prime}=H$. In a similar way, define $K^{\prime}$ and choose $\Gamma_{K}$ of finite index in $\Gamma$ such that $\Gamma_{K} \cap K^{\prime}=K$. Let $\Gamma_{0}=\Gamma_{H} \cap \Gamma_{K}$ and set $H_{0}=H \cap \Gamma_{0}$ and $K_{0}=K \cap \Gamma_{0}$. Then $\Gamma_{0}$ is a subgroup of finite index in $\Gamma, H_{0}=\Gamma_{0} \cap H^{\prime}$ and $K_{0}=\Gamma_{0} \cap K^{\prime}$. By Proposition 3.1 , by replacing $\Gamma$ with $\Gamma_{0}$, if necessary, we may assume that rank-one elements of $\{H, K\}$ are maximal cyclic subgroups of $\Gamma$ and rank-two elements of $\{H, K\}$ are maximal abelian subgroups of $\Gamma$. This assumption will be used if $H$ and/or $K$ is parabolic. However, the proof does not require loxodromic subgroups to be maximal.

Given the assumptions and reductions above, we need to consider the following cases.

Case 1. $H$ loxodromic, $K$ loxodromic

The group of orientation preserving isometries of $\mathbb{H}^{3}$ may be identified with $\operatorname{PSL}(2, \mathbb{C})$. Thus there exists a discrete, faithful representation $\rho: \pi_{1}(M) \rightarrow$ $\operatorname{PSL}(2, \mathbb{C})$ which is well-defined up to conjugation in $\operatorname{PSL}(2, \mathbb{C})$. Let $\mathbb{Q}(\operatorname{tr} \Gamma)$ denote the field obtained by adjoining the traces of the elements of $\rho(\Gamma)$ to $\mathbb{Q}$. Since $M$ has finite volume, it follows from Mostow Rigidity that $\mathbb{Q}(\operatorname{tr} \Gamma)$ is a number field. By Proposition 2.2(e) of [5] we may conjugate $\rho(\Gamma)$ in $\operatorname{PSL}(2, \mathbb{C})$ to lie in a finite field extension of $\mathbb{Q}(\operatorname{tr} \Gamma)$. Therefore we may view $\Gamma \subset \operatorname{PSL}(2, k)$, where $k$ is a finite extension of $\mathbb{Q}$. Write $H=\langle h\rangle$ and $K=\langle k\rangle$. In this case, $h$ and $k$ are conjugate in $\operatorname{PSL}(2, \mathbb{C})$ to matrices of the form

$$
\pm\left(\begin{array}{cc}
\lambda & 0 \\
0 & \lambda^{-1}
\end{array}\right), \lambda \in \mathbb{C},|\lambda| \neq 1, \text { and } \pm\left(\begin{array}{cc}
\omega & 0 \\
0 & \omega^{-1}
\end{array}\right), \omega \in \mathbb{C},|\omega| \neq 1
$$

respectively. Since $\mathbb{Q}(\operatorname{tr} \Gamma)$ is a number field, the eigenvalues, $\lambda$ and $\omega$, are algebraic numbers. By adjoining $\lambda$ and $\omega$ to $k$, if necessary, we may assume that $h$ and $k$ are diagonalizable over $k$. Therefore, after conjugating in $\operatorname{PSL}(2, k)$,

$$
h= \pm\left(\begin{array}{cc}
\lambda & 0 \\
0 & \lambda^{-1}
\end{array}\right) \text { and } k= \pm\left(\begin{array}{ll}
a & b \\
c & d
\end{array}\right)\left(\begin{array}{cc}
\omega & 0 \\
0 & \omega^{-1}
\end{array}\right)\left(\begin{array}{ll}
a & b \\
c & d
\end{array}\right)^{-1}
$$

for some

$$
g= \pm\left(\begin{array}{ll}
a & b \\
c & d
\end{array}\right) \in \operatorname{PSL}(2, k)
$$


By assumption, $h$ and $k$ do not commute. Since $\Gamma$ is discrete, it follows that the fixed points of $h$ on the sphere at infinity are disjoint from the fixed points of $k$. Therefore, all of the elements in $\{a, b, c, d\}$ are non-zero.

Let $G$ be the subgroup of $\operatorname{PSL}(2, k)$ generated by $\Gamma$ and $g$. We will show that $H K g$ is separable in $G$. Since the profinite topology on $G$ is equivariant under left and right multiplication, it will follow that $H K$ is separable in $G$, implying that $H K$ is separable in $\Gamma$, as required. To prove that $H K g$ is separable in $G$, note that

$$
\begin{gathered}
H K g=\left\{ \pm\left(\begin{array}{cc}
\lambda^{m} & 0 \\
0 & \lambda^{-m}
\end{array}\right)\left(\begin{array}{ll}
a & b \\
c & d
\end{array}\right)\left(\begin{array}{cc}
\omega^{n} & 0 \\
0 & \omega^{-n}
\end{array}\right)\right. \\
\left.= \pm\left(\begin{array}{cc}
a \lambda^{m} \omega^{n} & b \lambda^{m} \omega^{-n} \\
c \lambda^{-m} \omega^{n} & d \lambda^{-m} \omega^{-n}
\end{array}\right) \mid m, n \in \mathbb{Z}\right\},
\end{gathered}
$$

and let

$$
\gamma= \pm\left(\begin{array}{ll}
r & s \\
t & u
\end{array}\right) \in G-H K g
$$

be given. Since $M$ has finite volume, $\Gamma$ is finitely generated. Therefore, $G$ is finitely generated. Let $R$ be the ring generated by the coefficients of $G$ over $\mathbb{Z}$. Then $G \subset \operatorname{PSL}(2, R) \subset \operatorname{PSL}(2, k)$. Suppose that $\mathrm{rs} / \mathrm{ab}$ is not a multiplicative power of $\lambda^{2}$. Then by Corollary 2.5, there exist a finite ring $S$ and a ring homomorphism $\eta: R \rightarrow S$ such that $\eta(a b) \neq 0$ and $\eta(\mathrm{rs} / \mathrm{ab})$ is not a multiplicative power of $\eta\left(\lambda^{2}\right)$. The map $\eta$ induces a group homomorphism

$$
\bar{\eta}: G \hookrightarrow \operatorname{PSL}(2, R) \rightarrow \operatorname{PSL}(2, S) .
$$

Suppose that $\bar{\eta}(\gamma) \in \bar{\eta}(H K g)$. Then there exist elements $m, n \in \mathbb{Z}$ such that $\bar{\eta}(\gamma)=\bar{\eta}\left(h^{m} k^{n} g\right)$. Equating coefficients, we have:

$$
\begin{gathered}
\left\{\begin{array}{cc}
\eta(r)=\eta\left(a \lambda^{m} \omega^{n}\right), & \eta(s)=\eta\left(b \lambda^{m} \omega^{-n}\right), \\
\eta(t)=\eta\left(c \lambda^{-m} \omega^{n}\right), & \eta(u)=\eta\left(d \lambda^{-m} \omega^{-n}\right)
\end{array}\right\} \\
\text { or } \\
\left\{\begin{array}{cl}
\eta(-r)=\eta\left(a \lambda^{m} \omega^{n}\right), & \eta(-s)=\eta\left(b \lambda^{m} \omega^{-n}\right), \\
\eta(-t)=\eta\left(c \lambda^{-m} \omega^{n}\right), & \eta(-u)=\eta\left(d \lambda^{-m} \omega^{-n}\right)
\end{array}\right\} .
\end{gathered}
$$

In either case, $\eta(\mathrm{rs})=\eta\left(\mathrm{ab} \lambda^{2 m}\right)$, a contradiction. Therefore, we may assume that $\mathrm{rs} / \mathrm{ab}=\lambda^{2 m_{0}}$, for some $m_{0} \in \mathbb{Z}$. By a similar argument, we may assume that $\mathrm{rt} / \mathrm{ac}=\omega^{2 n_{0}}$, for some $n_{0} \in \mathbb{Z}$.

By assumption, $\gamma \notin H K g$. In particular,

$$
\left(\begin{array}{cc}
r & s \\
t & u
\end{array}\right) \notin\left\{ \pm\left(\begin{array}{cc}
a \lambda^{m_{0}} \omega^{n_{0}} & b \lambda^{m_{0}} \omega^{-n_{0}} \\
c \lambda^{-m_{0}} \omega^{n_{0}} & d \lambda^{-m_{0}} \omega^{-n_{0}}
\end{array}\right)\right\} .
$$

Since $R$ is finitely generated, $R \subset \mathcal{O}_{\mathrm{k}_{\mathfrak{p}}}$ for all but finitely many prime ideals $\mathfrak{p}$ of $\mathcal{O}_{\mathrm{k}}$. For each of these primes $\mathfrak{p}$ the residue class field map $\eta_{\mathfrak{p}}: \mathcal{O}_{\mathrm{k}_{\mathfrak{p}}} \rightarrow F_{\mathfrak{p}}$ induces a group homomorphism

$$
\bar{\eta}_{\mathfrak{p}}: G \hookrightarrow \operatorname{PSL}\left(2, \mathcal{O}_{\mathrm{k}_{\mathfrak{p}}}\right) \rightarrow \operatorname{PSL}\left(2, F_{\mathfrak{p}}\right)
$$


where $F_{\mathfrak{p}}$ is the residue class field of $\mathcal{O}_{\mathrm{k}_{\mathfrak{p}}}$. Choose $\mathfrak{p}$ such that

$$
(*) \quad\left(\begin{array}{cc}
\eta_{\mathfrak{p}}(r) & \eta_{\mathfrak{p}}(s) \\
\eta_{\mathfrak{p}}(t) & \eta_{\mathfrak{p}}(u)
\end{array}\right) \notin\left\{ \pm\left(\begin{array}{cc}
\eta_{\mathfrak{p}}\left(a \lambda^{m_{0}} \omega^{n_{0}}\right) & \eta_{\mathfrak{p}}\left(b \lambda^{m_{0}} \omega^{-n_{0}}\right) \\
\eta_{\mathfrak{p}}\left(c \lambda^{-m_{0}} \omega^{n_{0}}\right) & \eta_{\mathfrak{p}}\left(d \lambda^{-m_{0}} \omega^{-n_{0}}\right)
\end{array}\right)\right\} .
$$

Suppose $\bar{\eta}_{\mathfrak{p}}(\gamma) \in \bar{\eta}_{\mathfrak{p}}(H K g)$. Then there exist elements $m, n \in \mathbb{Z}$ such that $\bar{\eta}_{\mathfrak{p}}(\gamma)=\bar{\eta}_{\mathfrak{p}}\left(h^{m} k^{n} g\right)$. Equating coefficients as above, $\eta_{\mathfrak{p}}\left(\lambda^{2 m}\right)=\eta_{\mathfrak{p}}(\mathrm{rs} / \mathrm{ab})=$ $\eta_{\mathfrak{p}}\left(\lambda^{2 m_{0}}\right)$ and $\eta_{\mathfrak{p}}\left(\omega^{2 n}\right)=\eta_{\mathfrak{p}}(\mathrm{rt} / \mathrm{ac})=\eta_{\mathfrak{p}}\left(\omega^{2 n_{0}}\right)$. Therefore, $\eta_{\mathfrak{p}}\left(\lambda^{m}\right)= \pm \eta_{\mathfrak{p}}\left(\lambda^{m_{0}}\right)$ and $\eta_{\mathfrak{p}}\left(\omega^{n}\right)= \pm \eta_{\mathfrak{p}}\left(\omega^{n_{0}}\right)$, contradicting $(*)$. This completes the proof in this case.

Case 2. $H$ loxodromic, $K$ maximal parabolic

The proof of this case follows from the proof of Lemma 3.2 in [15].

Case 3. $H$ maximal parabolic, $K$ maximal parabolic

Since $M$ is a hyperbolic 3-manifold of finite volume, we may view $M$ as the interior of a compact manifold $M^{\prime}$ with a finite number of tori boundary components. Such a manifold can be obtained from $M$ by truncating the cusp tori. Furthermore, $\pi_{1}\left(M^{\prime}\right) \cong \pi_{1}(M)=\Gamma$. There is a one-to-one correspondence between the boundary tori of $M^{\prime}$ and the conjugacy classes of maximal parabolic subgroups of $\Gamma$. Suppose that $H$ and $K$ correspond to the same boundary component of $M^{\prime}$. Then there exists an element $\zeta \in \Gamma$ such that $K=\zeta H \zeta^{-1}$. Since $H$ and $K$ do not commute, $\zeta \notin H$. Since $H$ is a separable subgroup of $\Gamma$ 22. there exist a finite group $G$ and a group homomorphism $f: \Gamma \rightarrow G$ such that $f(\zeta) \notin f(H)$. Let $\Gamma_{0}$ be kernel of $f$, and set $H_{0}=H \cap \Gamma_{0}$ and $K_{0}=K \cap \Gamma_{0}$. Since $\Gamma_{0}$ is normal in $\Gamma, K_{0}=\zeta H_{0} \zeta^{-1}$. If there exists an element $v \in \Gamma_{0}$ such that $K_{0}=v H_{0} v^{-1}$, then $\left(v^{-1} \zeta\right) H_{0}\left(v^{-1} \zeta\right)^{-1}=H_{0}$. Therefore, $v^{-1} \zeta$ fixes the parabolic fixed point of $H$. Since $H$ is a maximal parabolic subgroup of $\Gamma$, $v^{-1} \zeta \in H$. But then $f(\zeta) \in f(H)$, a contradiction. We conclude that $H_{0}$ and $K_{0}$ are not conjugate in $\Gamma_{0}$. By Proposition 3.1 if $H_{0} K_{0}$ is separable in $\Gamma_{0}$, then $H K$ is separable in $\Gamma$. Therefore, by replacing $\Gamma$ with $\Gamma_{0}$, if necessary, we may assume that $H$ and $K$ correspond to different boundary components of $M^{\prime}$. Let $T_{1}$ and $T_{2}$ denote the boundary components of $M^{\prime}$ corresponding to $H$ and $K$, respectively. By Thurston's Hyperbolic Dehn Surgery Theorem [6], we may choose generators $h_{1}, h_{2}$ for $H$ such that $M_{h_{1}}$ and $M_{h_{2}}$ are complete hyperbolic 3-manifolds of finite volume, where $M_{h_{1}}$ and $M_{h_{2}}$ are the manifolds obtained by Dehn surgery on $M^{\prime}$ along $T_{1}$ sending $h_{1}$ and $h_{2}$, respectively, to a meridian of the attached solid torus. Similarly, we may choose generators $k_{1}, k_{2}$ for $K$ such that $M_{k_{1}}$ and $M_{k_{2}}$ are complete hyperbolic 3-manifolds of finite volume, where $M_{k_{1}}$ and $M_{k_{2}}$ are obtained by Dehn surgery on $M^{\prime}$ along $T_{2}$.

For simplicity we use the fact that the representation $\rho: \Gamma \rightarrow \operatorname{PSL}(2, \mathbb{C})$ may be lifted to a representation

$$
\Gamma \rightarrow \mathrm{SL}(2, \mathbb{C}) .
$$


(See Proposition 3.1.1 of [12.) Therefore, we view $\Gamma \subset \mathrm{SL}(2, \mathbb{C})$. By an argument similar to that in Case 1 , we may assume that $\Gamma \subset \mathrm{SL}(2, k)$, where $k$ is a finite field extension of $\mathbb{Q}$,

$$
h_{1}=\left(\begin{array}{ll}
1 & 1 \\
0 & 1
\end{array}\right) \text { and } h_{2}=\left(\begin{array}{cc}
1 & \beta_{1} \\
0 & 1
\end{array}\right),
$$

for a fixed element $\beta_{1} \in \mathbb{C}-\mathbb{R}$. If $A_{1}=\left\{m+n \beta_{1} \mid m, n \in \mathbb{Z}\right\}$, then

$$
H=\left\{\left(\begin{array}{ll}
1 & x \\
0 & 1
\end{array}\right) \mid x \in A_{1}\right\}
$$

Moreover,

$k_{1}=\left(\begin{array}{ll}a_{1} & b_{1} \\ c_{1} & d_{1}\end{array}\right)\left(\begin{array}{ll}1 & 1 \\ 0 & 1\end{array}\right)\left(\begin{array}{ll}a_{1} & b_{1} \\ c_{1} & d_{1}\end{array}\right)^{-1}$ and $k_{2}=\left(\begin{array}{ll}a_{1} & b_{1} \\ c_{1} & d_{1}\end{array}\right)\left(\begin{array}{cc}1 & \beta_{2} \\ 0 & 1\end{array}\right)\left(\begin{array}{ll}a_{1} & b_{1} \\ c_{1} & d_{1}\end{array}\right)^{-1}$

for fixed elements

$$
\left(\begin{array}{ll}
a_{1} & b_{1} \\
c_{1} & d_{1}
\end{array}\right) \in \mathrm{SL}(2, \mathbb{C}) \text { and } \beta_{2} \in \mathbb{C}-\mathbb{R} .
$$

If $A_{2}=\left\{m+n \beta_{2} \mid m, n \in \mathbb{Z}\right\}$, then

$$
K=\left\{\left(\begin{array}{cc}
1-y a_{1} c_{1} & y a_{1}^{2} \\
-y c_{1}^{2} & 1+y a_{1} c_{1}
\end{array}\right) \mid y \in A_{2}\right\} .
$$

Since $H$ and $K$ do not commute, $c_{1} \neq 0$. Note that $a_{1}{ }^{2}$ and $c_{1}{ }^{2}$ are elements of the coefficient field $k$. Since $k$ is a number field, $a_{1}$ and $c_{1}$ are algebraic numbers. Therefore, after adjoining $a_{1}$ and $c_{1}$ to $k$, and conjugating $\Gamma$ in $\operatorname{SL}(2, k)$ by

$$
\left(\begin{array}{cc}
1 & a_{1} / c_{1} \\
0 & 1
\end{array}\right)
$$

we may assume that

$$
\begin{gathered}
H=\left\{\left(\begin{array}{cc}
1 & -a_{1} / c_{1} \\
0 & 1
\end{array}\right)\left(\begin{array}{cc}
1 & x \\
0 & 1
\end{array}\right)\left(\begin{array}{cc}
1 & a_{1} / c_{1} \\
0 & 1
\end{array}\right)=\left(\begin{array}{cc}
1 & x \\
0 & 1
\end{array}\right) \mid x \in A_{1}\right\}, \text { and } \\
K=\left\{\left(\begin{array}{cc}
1 & -a_{1} / c_{1} \\
0 & 1
\end{array}\right)\left(\begin{array}{cc}
1-y a_{1} c_{1} & y a_{1}^{2} \\
-y c_{1}^{2} & 1+y a_{1} c_{1}
\end{array}\right)\left(\begin{array}{cc}
1 & a_{1} / c_{1} \\
0 & 1
\end{array}\right)=\left(\begin{array}{cc}
1 & 0 \\
-y c_{1}{ }^{2} & 1
\end{array}\right) \mid y \in A_{2}\right\} .
\end{gathered}
$$

Then

$$
H K=\left\{\left(\begin{array}{ll}
1 & x \\
0 & 1
\end{array}\right)\left(\begin{array}{cc}
1 & 0 \\
-y c_{1}{ }^{2} & 1
\end{array}\right)=\left(\begin{array}{cc}
1-x y c_{1}^{2} & x \\
-y c_{1}^{2} & 1
\end{array}\right) \mid x \in A_{1}, y \in A_{2}\right\} .
$$

To show that $H K$ is separable in $\Gamma$, let

$$
\gamma=\left(\begin{array}{ll}
r & s \\
t & u
\end{array}\right) \in \Gamma-H K
$$


be given. Suppose that $u \neq 1$. As in Case 1 , there exists a finitely generated ring $R_{1}$ such that $\Gamma \subset \mathrm{SL}\left(2, R_{1}\right) \subset \mathrm{SL}(2, k)$. Since $R_{1}$ is finitely generated, $R_{1} \subset \mathcal{O}_{\mathrm{k}_{\mathfrak{p}}}$ for all but finitely many primes $\mathfrak{p}$ of $\mathcal{O}_{\mathrm{k}_{\mathfrak{p}}}$. For each of these primes $\mathfrak{p}$ the residue class field map $\eta_{\mathfrak{p}}: \mathcal{O}_{\mathfrak{k}_{\mathfrak{p}}} \rightarrow F_{\mathfrak{p}}$ induces a group homomorphism

$$
\bar{\eta}_{\mathfrak{p}}: \Gamma \hookrightarrow \mathrm{SL}\left(2, \mathcal{O}_{\mathfrak{k}_{\mathfrak{p}}}\right) \rightarrow \mathrm{SL}\left(2, F_{\mathfrak{p}}\right)
$$

where $F_{\mathfrak{p}}$ is the residue class field of $\mathcal{O}_{\mathrm{k}_{\mathfrak{p}}}$. Choose $\mathfrak{p}$ such that $\eta_{\mathfrak{p}}(u) \neq \eta_{\mathfrak{p}}(1)$. Then $\bar{\eta}_{\mathfrak{p}}(\gamma) \notin \bar{\eta}_{\mathfrak{p}}(H K)$, as required. Therefore, we may assume that $u=1$. Since the determinant of $\gamma$ is equal to 1 ,

$$
\gamma=\left(\begin{array}{cc}
1+s t & s \\
t & 1
\end{array}\right)
$$

Let $B_{1}=\left\{m+n \beta_{1} \mid m, n \in \mathbb{Q}\right\}$ and $B_{2}=\left\{m+n \beta_{2} \mid m, n \in \mathbb{Q}\right\}$. Suppose that either $s \notin B_{1}$ or $-t / c_{1}^{2} \notin B_{2}$. By Theorem 2.6, there exist a finite ring $S$ and a ring homomorphism $\eta: R_{1} \rightarrow S$ such that $\eta(s) \notin \eta\left(B_{1}\right)$ or $\eta\left(-t / c_{1}^{2}\right) \notin \eta\left(B_{2}\right)$, respectively. This ring homomorphism induces a group homomorphism

$$
\bar{\eta}: \Gamma \hookrightarrow \mathrm{SL}\left(2, R_{1}\right) \rightarrow \mathrm{SL}(2, S),
$$

such that $\bar{\eta}(\gamma) \notin \bar{\eta}(H K)$. Therefore, we may assume that $s \in B_{1}$ and $-t / c_{1}^{2} \in$ $B_{2}$. Moreover, since $\gamma \notin H K$, either $s \notin A_{1}$ or $-t / c_{1}^{2} \notin A_{2}$. We will assume that $s \notin A_{1}$. (The argument if $-t / c_{1}^{2} \notin A_{2}$ is similar, with the roles of $H$ and $K$ interchanged). Since $s \in B_{1}$, there exists a non-zero integer $v_{0}$ such that $v_{0} s \in A_{1}$. Write $v_{0} s=m_{0}+n_{0} \beta_{1}$, where $m_{0}, n_{0} \in \mathbb{Z}$. Since $s \notin A_{1}$, either $v_{0}$ does not divide $m_{0}$ or $v_{0}$ does not divide $n_{0}$. We will assume that $v_{0}$ does not divide $m_{0}$. (The argument if $v_{0}$ does not divide $n_{0}$ is similar, with the roles of $h_{1}$ and $h_{2}$ interchanged). Let $M_{h_{2}}$ be the hyperbolic 3-manifold obtained from $M^{\prime}$ by Dehn surgery along $T_{1}$, as defined above. Let

$$
\phi: \Gamma \cong \pi_{1}\left(M^{\prime}\right) \rightarrow \pi_{1}\left(M_{h_{2}}\right)
$$

be the homomorphism induced by inclusion. Then $\phi(H)$ is an infinite cyclic loxodromic subgroup of $\pi_{1}\left(M_{h_{2}}\right)$ generated by $\phi\left(h_{1}\right)$, and $\phi\left(h_{2}\right)$ is trivial. By assumption, $H$ and $K$ correspond to different boundary components of $M^{\prime}$. Therefore, $\phi(K)$ is a maximal parabolic subgroup of $\pi_{1}\left(M_{h_{2}}\right)$. If $\phi(\gamma) \notin \phi(H K)$, then we are done by Case 2. Therefore, we may assume that $\phi(\gamma)=\phi\left(h_{1}^{m_{1}}\right) \phi\left(k_{\gamma}\right)$, for some $m_{1} \in \mathbb{Z}$ and $k_{\gamma} \in K$. Since $v_{0}$ does not divide $m_{0}, v_{0} m_{1}-m_{0} \neq 0$.

Recall that $\Gamma \subset \mathrm{SL}\left(2, R_{1}\right) \subset \mathrm{SL}(2, k)$. Let $L$ denote the normal closure of $k$ over $\mathbb{Q}$ and let $\tau \in \operatorname{Gal}(L / \mathbb{Q})$ represent complex conjugation. Since $\beta_{1} \in \mathbb{C}-\mathbb{R}$, $\tau\left(\beta_{1}\right) \neq \beta_{1}$. By the Tchebotarev Density Theorem, there exist infinitely many primes $p$ of $\mathbb{Q}$ with unramified extension $\mathfrak{p}$ in $L$ such that $\tau$ is the Frobenius automorphism for $\mathfrak{p} / p$. Fix one such $\mathfrak{p} / p$ such that $p$ is an odd prime, $p$ does not divide $v_{0} m_{1}-m_{0}$ and $R_{1} \subset \mathcal{O}_{\mathrm{L}_{\mathfrak{p}}}$, where $\mathcal{O}_{\mathrm{L}_{\mathfrak{p}}}$ denotes the ring of integers in the $\mathfrak{p}$-adic field $L_{\mathfrak{p}}$. Let $F_{\mathfrak{p}}$ denote the residue class field of $\mathcal{O}_{L_{\mathfrak{p}}}$ and let $\mathbb{F}_{p}$ denote the finite field of $p$ elements. Let $\eta_{1}$ be the composition of the inclusion map of $R_{1}$ into $\mathcal{O}_{\mathrm{L}_{\mathfrak{p}}}$ with the residue map:

$$
\eta_{1}: R_{1} \hookrightarrow \mathcal{O}_{\mathrm{L}_{\mathfrak{p}}} \rightarrow F_{\mathfrak{p}}
$$


Since $\tau$ is the Frobenius automorphism of $L / \mathbb{Q}$ with respect to $\mathfrak{p} / p, \operatorname{Gal}\left(L_{\mathfrak{p}} / \mathbb{Q}_{p}\right)=$ $\left\langle\tau^{\prime}\right\rangle$ where $\tau^{\prime}=\tau$ on $L$. Since $\tau\left(\beta_{1}\right) \neq \beta_{1}, \beta_{1} \notin \mathbb{Q}_{p}$. The Galois group of $F_{\mathfrak{p}} / \mathbb{F}_{p}$ is also induced by $\tau$. It follows that $\eta_{1}\left(\beta_{1}\right) \notin \mathbb{F}_{p}$. The map $\eta_{1}: R_{1} \rightarrow F_{\mathfrak{p}}$ induces a group homomorphism

$$
\psi_{1}: \Gamma \hookrightarrow \mathrm{SL}\left(2, R_{1}\right) \rightarrow \mathrm{SL}\left(2, F_{\mathfrak{p}}\right) .
$$

If $\psi_{1}(\gamma) \notin \psi_{1}(H K)$, then we are done. Suppose that $\psi_{1}(\gamma)=\psi_{1}(h k)$ for some $h \in H, k \in K$. If $h=h_{1}^{m} h_{2}^{n}$ and $x=m+n \beta_{1}$, then

$$
\left(\begin{array}{cc}
\eta_{1}(1+s t) & \eta_{1}(s) \\
\eta_{1}(t) & 1
\end{array}\right)=\psi_{1}(\gamma)=\psi_{1}(h k)=\left(\begin{array}{cc}
\eta_{1}\left(1-x y c_{1}^{2}\right) & \eta_{1}(x) \\
\eta_{1}\left(-y c_{1}^{2}\right) & 1
\end{array}\right)
$$

for some $y \in A_{2}$ corresponding to $k$. Therefore, $\eta_{1}\left(v_{0} m+v_{0} n \beta_{1}\right)=\eta_{1}\left(v_{0} x\right)=$ $\eta_{1}\left(v_{0} s\right)=\eta_{1}\left(m_{0}+n_{0} \beta_{1}\right)$. Since $\eta_{1}\left(\beta_{1}\right) \notin \mathbb{F}_{p}$, the set $\left\{1, \eta_{1}\left(\beta_{1}\right)\right\}$ is linearly independent over $\mathbb{F}_{p}$. It follows that

$$
\text { (*) } v_{0} m \equiv m_{0}(\bmod p) .
$$

Now consider the hyperbolic 3-manifold $M_{h_{2}}$. Recall that $\phi(H)$ is a loxodromic subgroup of $\pi_{1}\left(M_{h_{2}}\right)$ generated by $\phi\left(h_{1}\right), \phi(K)$ is a maximal parabolic subgroup of $\pi_{1}\left(M_{h_{2}}\right)$, and $\phi(\gamma)=\phi\left(h_{1}^{m_{1}} k_{\gamma}\right)$, for some $m_{1} \in \mathbb{Z}$ and $k_{\gamma} \in K$. As before, there exists a finitely generated ring $R_{2}$ in a number field $F$, such that $\pi_{1}\left(M_{h_{2}}\right) \subset \mathrm{SL}\left(2, R_{2}\right) \subset \mathrm{SL}(2, F)$. Moreover, after conjugating $\pi_{1}\left(M_{h_{2}}\right)$ in $\mathrm{SL}(2, F)$, if necessary, we may assume that

$$
\begin{gathered}
\phi\left(h_{1}\right)=\left(\begin{array}{cc}
\lambda & 0 \\
0 & \lambda^{-1}
\end{array}\right) \text { and } \\
\phi(K)=\left\{\left(\begin{array}{ll}
a_{2} & b_{2} \\
c_{2} & d_{2}
\end{array}\right)\left(\begin{array}{ll}
1 & z \\
0 & 1
\end{array}\right)\left(\begin{array}{ll}
a_{2} & b_{2} \\
c_{2} & d_{2}
\end{array}\right)^{-1} \mid z \in A_{3}=\left\{m+n \beta_{3} \mid m, n \in \mathbb{Z}\right\}\right\},
\end{gathered}
$$

for some

$$
\left(\begin{array}{ll}
a_{2} & b_{2} \\
c_{2} & d_{2}
\end{array}\right) \in \mathrm{SL}(2, F) \text { and } \beta_{3} \in \mathbb{C}-\mathbb{R}
$$

Then

$$
\phi(H K)=\left\{\left(\begin{array}{cc}
\lambda^{m}\left(1-a_{2} c_{2} z\right) & \lambda^{m} a_{2}^{2} z \\
-\lambda^{-m} c_{2}^{2} z & \lambda^{-m}\left(1+a_{2} c_{2} z\right)
\end{array}\right) \mid m \in \mathbb{Z}, z \in A_{3}\right\} .
$$

Write

$$
\phi\left(k_{\gamma}\right)=\left(\begin{array}{cc}
1 & z_{\gamma} \\
0 & 1
\end{array}\right), z_{\gamma} \in A_{3} .
$$

Since $\pi_{1}\left(M_{h_{2}}\right)$ is discrete, $\phi(H)$ and $\phi(K)$ do not share a fixed point. Therefore, $a_{2}$ and $c_{2}$ are non-zero. By Corollary 2.3. there exist a finite field $S$ and a ring homomorphism $\eta_{2}: R_{2} \rightarrow S$ such that the multiplicative order of $\eta_{2}(\lambda)$ is divisible by $p$, and $\eta_{2}\left(a_{2}\right)$ and $\eta_{2}\left(c_{2}\right)$ are non-zero in $S$. Since $\lambda$ is a unit in 
$R_{2}, \eta_{2}(\lambda) \neq 0$. Let $o$ denote the multiplicative order of $\eta_{2}(\lambda)$. Consider the composition:

$$
\psi_{2}: \Gamma \rightarrow \pi_{1}\left(M_{h_{2}}\right) \hookrightarrow \mathrm{SL}\left(2, R_{2}\right) \rightarrow \mathrm{SL}(2, S),
$$

where the first map $\phi: \Gamma \rightarrow \pi_{1}\left(M_{h_{2}}\right)$ is induced by inclusion and the last map $\mathrm{SL}\left(2, R_{2}\right) \rightarrow \mathrm{SL}(2, S)$ is induced by $\eta_{2}$. If $\psi_{2}(\gamma) \notin \psi_{2}(H K)$, then we are done. Suppose that $\psi_{2}(\gamma)=\psi_{2}(h k)$, for some $h \in H, k \in K$. If $h=h_{1}^{m} h_{2}^{n}$, then

$$
\begin{gathered}
\psi_{2}(\gamma)=\left(\begin{array}{cc}
\eta_{2}\left(\lambda^{m_{1}}\left(1-a_{2} c_{2} z_{\gamma}\right)\right) & \eta_{2}\left(\lambda^{m_{1}} a_{2}{ }^{2} z_{\gamma}\right) \\
\eta_{2}\left(-\lambda^{-m_{1}} c_{2}{ }^{2} z_{\gamma}\right) & \eta_{2}\left(\lambda^{-m_{1}}\left(1+a_{2} c_{2} z_{\gamma}\right)\right)
\end{array}\right)= \\
\psi_{2}(h k)=\left(\begin{array}{cc}
\eta_{2}\left(\lambda^{m}\left(1-a_{2} c_{2} z\right)\right) & \eta_{2}\left(\lambda^{m} a_{2}{ }^{2} z\right) \\
\eta_{2}\left(-\lambda^{-m} c_{2}{ }^{2} z\right) & \eta_{2}\left(\lambda^{-m}\left(1+a_{2} c_{2} z\right)\right)
\end{array}\right),
\end{gathered}
$$

for some $a \in A_{3}$ corresponding to $k$. This gives the equations:

$$
\begin{aligned}
\eta_{2}\left(\lambda^{m_{1}}\left(1-a_{2} c_{2} z_{\gamma}\right)\right) & =\eta_{2}\left(\lambda^{m}\left(1-a_{2} c_{2} z\right)\right) \\
\eta_{2}\left(\lambda^{m_{1}} a_{2}{ }^{2} z_{\gamma}\right) & =\eta_{2}\left(\lambda^{m} a_{2}{ }^{2} z\right) \\
\eta_{2}\left(-\lambda^{-m_{1}} c_{2}{ }^{2} z_{\gamma}\right) & =\eta_{2}\left(-\lambda^{-m} c_{2}{ }^{2} z\right) \\
\eta_{2}\left(\lambda^{-m_{1}}\left(1+a_{2} c_{2} z_{\gamma}\right)\right) & =\eta_{2}\left(\lambda^{-m}\left(1+a_{2} c_{2} z\right)\right) .
\end{aligned}
$$

If $\eta_{2}\left(z_{\gamma}\right)=0$, then $\eta_{2}(z)=0$, and so $\eta_{2}\left(\lambda^{m}\right)=\eta_{2}\left(\lambda^{m_{1}}\right)$. If $\eta_{2}\left(z_{\gamma}\right) \neq 0$, then by solving for $\eta_{2}\left(z / z_{\gamma}\right)$ in the second and third equations, we have that $\eta_{2}\left(\lambda^{2 m}\right)=\eta_{2}\left(\lambda^{2 m_{1}}\right)$. In either case, $2 m \equiv 2 m_{1}(\bmod o)$. Since $p$ divides $o$ and $p$ is an odd prime, it follows that

$$
(* *) \quad m \equiv m_{1}(\bmod p) .
$$

Finally, consider the product

$$
\left(\psi_{1} \times \psi_{2}\right): \Gamma \rightarrow \mathrm{SL}\left(2, F_{\mathfrak{p}}\right) \times \mathrm{SL}(2, S) .
$$

If $\left(\psi_{1} \times \psi_{2}\right)(\gamma) \notin\left(\psi_{1} \times \psi_{2}\right)(H K)$, then we are done. Suppose that $\left(\psi_{1} \times\right.$ $\left.\psi_{2}\right)(\gamma)=\left(\psi_{1} \times \psi_{2}\right)(h k)$ for some $h \in H, k \in K$. Then $\psi_{1}(\gamma)=\psi_{1}(h k)$ and $\psi_{2}(\gamma)=\psi_{2}(h k)$. If $h=h_{1}^{m} h_{2}^{n}$, then by $(*)$ and $(* *), v_{0} m \equiv m_{0}(\bmod p)$ and $m \equiv m_{1}(\bmod p)$. Therefore, $v_{0} m_{1} \equiv m_{0}(\bmod p)$. This contradicts the fact that we chose $p$ not to divide $v_{0} m_{1}-v_{0}$. This completes the proof of Case 3 .

Case 4. $H$ loxodromic, $K$ parabolic

Let $K^{\prime}$ be the maximal parabolic subgroup of $\Gamma$ containing $K$. By Case 2 and the assumptions at the beginning of the proof, we may assume that $K$ is a maximal cyclic subgroup of $\Gamma$. Therefore, there exists a basis $\left\{k_{1}, k_{2}\right\}$ of $K^{\prime}$ such that $K=\left\langle k_{1}\right\rangle$. Let $H=\langle h\rangle$. To prove that $H K$ is separable in $\Gamma$, let $\gamma \in \Gamma-H K$ be given. By Case 2, we may assume that $\gamma \in H K^{\prime}-H K$. Therefore, $\gamma=h^{a_{0}} k_{1}^{m_{0}} k_{2}^{n_{0}}$, for some $a_{0}, m_{0}, n_{0} \in \mathbb{Z}$. Since $\gamma \notin H K, n_{0} \neq 0$. As above, we may assume that $\Gamma \subset \mathrm{SL}(2, R) \subset \mathrm{SL}(2, k)$, where $R$ is a finitely generated ring contained in a number field $k$. Moreover,

$$
k_{1}=\left(\begin{array}{ll}
1 & 1 \\
0 & 1
\end{array}\right) \text { and } k_{2}=\left(\begin{array}{cc}
1 & \beta \\
0 & 1
\end{array}\right)
$$


for some $\beta \in \mathbb{C}-\mathbb{R}$. Since $H$ is loxodromic, $h$ is conjugate in $\operatorname{SL}(2, \mathbb{C})$ to an element

$$
f=\left(\begin{array}{cc}
\lambda & 0 \\
0 & \lambda^{-1}
\end{array}\right), \lambda \in \mathbb{C},|\lambda| \neq 1 .
$$

The eigenvalue $\lambda$ is an algebraic number. By adjoining $\lambda$ to $k$, we may assume that $h$ is diagonalizable over $\operatorname{SL}(2, k)$. Therefore,

$$
h=\left(\begin{array}{ll}
a & b \\
c & d
\end{array}\right)\left(\begin{array}{cc}
\lambda & 0 \\
0 & \lambda^{-1}
\end{array}\right)\left(\begin{array}{ll}
a & b \\
c & d
\end{array}\right)^{-1}, \text { for some }\left(\begin{array}{ll}
a & b \\
c & d
\end{array}\right) \in \operatorname{SL}(2, k) .
$$

By expanding $R$, if necessary, we may assume that $\left\{\lambda, \lambda^{-1}, a, b, c, d\right\} \subset R$. Let $L$ denote the normal closure of $k$ over $\mathbb{Q}$ and let $\tau \in \operatorname{Gal}(L / \mathbb{Q})$ represent complex conjugation. As in Case 3, by the Tchebotarev Density Theorem, there exist infinitely many primes $p$ of $\mathbb{Q}$ with unramified extension $\mathfrak{p}$ in $L$ such that $\tau$ is the Frobenius automorphism for $\mathfrak{p} / p$. Fix one such $\mathfrak{p} / p$ such that $p$ does not divide $n_{0}$ and $R \subset \mathcal{O}_{\mathrm{L}_{\mathfrak{p}}}$, where $\mathcal{O}_{\mathrm{L}_{\mathfrak{p}}}$ denotes the ring of integers in the $\mathfrak{p}$-adic field $L_{\mathfrak{p}}$. Let $F_{\mathfrak{p}}$ denote the residue class field of $\mathcal{O}_{\mathrm{L}_{\mathfrak{p}}}$ and let $\mathbb{F}_{p}$ denote the finite field of $p$ elements. Let $\eta$ be the composition of the inclusion map of $R$ into $\mathcal{O}_{\mathrm{L}_{\mathrm{p}}}$ with the residue map:

$$
\eta: R \hookrightarrow \mathcal{O}_{\mathrm{L}_{\mathfrak{p}}} \rightarrow F_{\mathfrak{p}}
$$

Since $\tau$ is the Frobenius automorphism of $L / \mathbb{Q}$ with respect to $\mathfrak{p} / p, \eta(\beta) \notin \mathbb{F}_{p}$. The map $\eta: R \rightarrow F_{\mathfrak{p}}$ induces a group homomorphism

$$
\psi: \Gamma \hookrightarrow \mathrm{SL}(2, R) \rightarrow \mathrm{SL}\left(2, F_{\mathfrak{p}}\right)
$$

Suppose that $\psi(\gamma) \in \psi(H K)$. Then $\psi\left(h^{a_{0}} k_{1}^{m_{0}} k_{2}^{n_{0}}\right)=\psi\left(h^{a} k_{1}^{m}\right)$, for some $a, m \in$ $\mathbb{Z}$. Therefore, $\psi\left(k_{1}^{m_{0}-m} k_{2}^{n_{0}}\right)=\psi\left(h^{a-a_{0}}\right)$. The trace of each element in $K^{\prime}$ is equal to 2. It follows that $\eta\left(\lambda^{a-a_{0}}\right)+1 / \eta\left(\lambda^{a-a_{0}}\right)=2$, and so $\eta\left(\lambda^{a-a_{0}}\right)=1$. This means that $\psi\left(f^{a-a_{0}}\right)$ is trivial. Since $h$ is conjugate to $f$ in $\operatorname{SL}(2, R)$, $\psi\left(h^{a-a_{0}}\right)=\psi\left(k_{1}^{m_{0}-m} k_{2}^{n_{0}}\right)$ is trivial. Therefore, $\eta\left(m_{0}-m+n_{0} \beta\right)=0$. Since $\eta(\beta) \notin \mathbb{F}_{p}$, the set $\{1, \eta(\beta)\}$ is linearly independent over $\mathbb{F}_{p}$. It follows that $\eta\left(n_{0}\right)=0$. But this contradicts the fact that $p$ does not divide $n_{0}$. We conclude that $\psi(\gamma) \notin \psi(H K)$, as required

Case 5. $H$ parabolic, $K$ parabolic

Let $H^{\prime}$ and $K^{\prime}$ be the maximal parabolic subgroups of $\Gamma$, containing $H$ and $K$, respectively. By the assumptions at the beginning of the proof, either $H=H^{\prime}$, or $H$ is a maximal cyclic subgroup of $H^{\prime}$. A similar statement is true for $K$. If $H=H^{\prime}$ and $K=K^{\prime}$ then we are done by Case 3 . Therefore, we assume that either $H$ or $K$ is infinite cyclic. Choose bases $\left\{h_{1}, h_{2}\right\}$ for $H^{\prime}$ and $\left\{k_{1}, k_{2}\right\}$ for $K^{\prime}$ such that $H=\left\langle h_{1}\right\rangle$ if $H$ is infinite cyclic, and $K=\left\langle k_{1}\right\rangle$ if $K$ is infinite cyclic. To show that $H K$ is separable in $\Gamma$, let $\gamma \in \Gamma-H K$ be given. By Case 3, we may assume that $\gamma \in H^{\prime} K^{\prime}-H K$. Therefore, $\gamma=h_{1}^{m_{1}} h_{2}^{n_{1}} k_{1}^{m_{2}} k_{2}^{n_{2}}$, for some $m_{1}, m_{2}, n_{1}, n_{2} \in \mathbb{Z}$. Since $\gamma \notin H K$, either $(i) H$ is infinite cyclic and $n_{1} \neq 0$ or $(i i) K$ is infinite cyclic and $n_{2} \neq 0$. Without loss of generality, assume that $(i)$ holds. 
The argument is then very similar to the argument in Case 4. We may assume that $\Gamma \subset \mathrm{SL}(2, R) \subset \mathrm{SL}(2, k)$, where $R$ is a finitely generated ring in a number field $k$. Moreover,

$$
\begin{gathered}
h_{1}=\left(\begin{array}{ll}
1 & 1 \\
0 & 1
\end{array}\right), h_{2}=\left(\begin{array}{cc}
1 & \beta_{1} \\
0 & 1
\end{array}\right), \text { and } \\
K=\left\{\left(\begin{array}{ll}
a & b \\
c & d
\end{array}\right)\left(\begin{array}{ll}
1 & y \\
0 & 1
\end{array}\right)\left(\begin{array}{ll}
a & b \\
c & d
\end{array}\right)^{-1}=\left(\begin{array}{cc}
1-a c y & a^{2} y \\
-c^{2} y & 1+a c y
\end{array}\right) \mid y \in\left\{m+n \beta_{2}\right\}\right\}, \\
\text { for some }\left(\begin{array}{ll}
a & b \\
c & d
\end{array}\right) \in \operatorname{SL}(2, R) \text { and } \beta_{1}, \beta_{2} \in \mathbb{C}-\mathbb{R} .
\end{gathered}
$$

By assumption, $H$ and $K$ do not commute. Therefore $c \neq 0$. As in Case 4 , by the Tchebotarev Density Theorem, there exist a prime $p$ that does not divide $n_{1}$, a finite field $F_{\mathfrak{p}}$ of characteristic $p$, and a ring homomorphism

$$
\eta: R \rightarrow F_{\mathfrak{p}}
$$

such that the set $\left\{1, \eta\left(\beta_{1}\right)\right\}$ is linearly independent over $\mathbb{F}_{p}$, and $\eta(c) \neq 0$. The map $\eta$ induces a group homomorphism

$$
\psi: \Gamma \hookrightarrow \mathrm{SL}(2, R) \rightarrow \mathrm{SL}\left(2, F_{\mathfrak{p}}\right) .
$$

Suppose that $\psi(\gamma) \in \psi(H K)$. Then $\psi\left(h_{1}^{m_{1}} h_{2}^{n_{1}} k_{1}^{m_{2}} k_{2}^{n_{2}}\right)=\psi\left(h_{1}^{u_{1}} k_{1}^{u_{2}} k_{2}^{v_{2}}\right)$, for some $u_{1}, u_{2}, v_{2} \in \mathbb{Z}$. Therefore, $\psi\left(h_{1}^{m_{1}-u_{1}} h_{2}^{n_{1}}\right)=\psi\left(k_{1}^{u_{2}-m_{2}} k_{2}^{v_{2}-n_{2}}\right)$. Since $H$ is upper triangular and $\eta(c) \neq 0$, it follows that $\psi\left(k_{1}^{u_{2}-m_{2}} k_{2}^{v_{2}-n_{2}}\right)=\psi\left(h_{1}^{m_{1}-u_{1}} h_{2}^{n_{1}}\right)$ is trivial. Therefore, $\eta\left(m_{1}-u_{1}+n_{1} \beta_{1}\right)=0$. Since $\left\{1, \eta\left(\beta_{1}\right)\right\}$ is linearly independent over $\mathbb{F}_{p}, \eta\left(n_{1}\right)=0$. But this contradicts the fact that $p$ does not divide $n_{1}$.

We conclude this section with some corollaries to the proof of Theorem 3.2 Since only Case 3 of Theorem 3.2 uses the full strength of the finite covolume assumption, it is natural to consider finitely generated Kleinian groups which are not necessarily of finite covolume.

Corollary 3.3. Let $\Gamma$ be a finitely generated, torsion-free Kleinian group. Given an abelian subgroup $G$ of $\Gamma$, let $A(G)$ denote the maximal abelian subgroup of $\Gamma$ containing $G$. Suppose that $H$ and $K$ are abelian subgroups of $\Gamma$ such that $A(H)$ and $A(K)$ are not both free abelian of rank 2. Then the double coset $H K$ is separable in $\Gamma$.

Proof. If $\Gamma$ is elementary, then $\Gamma$ is virtually abelian and hence the result follows from Proposition 3.1 and 3 . If $\Gamma$ is non-elementary, then $\Gamma$ is isomorphic to a geometrically finite Kleinian group $\Gamma_{1}$ such that $(i)$ every maximal parabolic subgroup of $\Gamma_{1}$ has rank 2 , and (ii) the traces of the elements of $\Gamma_{1}$ are algebraic numbers. (See Theorem 1 of $[3$, or Theorem 4.2 of [30].) By replacing $\Gamma$ with $\Gamma_{1}$, we assume that conditions $(i)$ and (ii) hold for $\Gamma$. Our assumptions then 
imply that at least one of $H$ or $K$ must be loxodromic. The proof then follows from Cases 1, 2 and 4 of Theorem 3.2 .

In Theorem 3.2 we prove that certain double cosets of Kleinian groups of finite covolume are closed in the profinite topology on $\Gamma$. We now consider the congruence topology on $\Gamma$.

Definition 3.4. Let $k$ be a number field and let $\Gamma$ be a finitely generated subgroup of $\operatorname{PSL}(2, k)$. Since $\Gamma$ is finitely generated, $\Gamma \subset \operatorname{PSL}(2, R) \subset \operatorname{PSL}(2, k)$, where $R$ is a ring obtained from $\mathcal{O}_{\mathrm{k}}$ by inverting a finite number of elements. This ring $R$ is Dedekind and, therefore, for any non-zero ideal $I$, the quotient $R / I$ is finite. The quotient map $R \rightarrow R / I$ induces a congruence homomorphism

$$
\eta: \Gamma \hookrightarrow \operatorname{PSL}(2, R) \rightarrow \operatorname{PSL}(2, R / I) .
$$

We say that a subset $X$ of $\Gamma$ is closed in the congruence topology on $\Gamma$ if for every element $\gamma \in \Gamma-X$, there exists a congruence homomorphism $\eta$ such that $\eta(\gamma) \notin \eta(X)$.

Recall that a set $X \subset \Gamma$ is closed in the profinite topology on $\Gamma$ if for every element $\gamma \in \Gamma-X$, there exist a finite group $G$ and a group homomorphism $\phi$ : $\Gamma \rightarrow G$ such that $\phi(\gamma) \notin \phi(X)$. For the profinite topology we consider all group homomorphisms from $\Gamma$ into finite groups. For the congruence topology we consider only congruence homomorphisms. Therefore, the congruence topology is weaker than the profinite topology.

Corollary 3.5. Let $M=\mathbb{H}^{3} / \Gamma$ be an orientable hyperbolic 3-orbifold of finite volume. Let $\rho: \Gamma \rightarrow \operatorname{PSL}(2, \mathbb{C})$ be a discrete, faithful representation such that the coefficient field of $\rho(\Gamma)$ is a finite field extension of $\mathbb{Q}$. Then we may view $\rho(\Gamma) \subset \operatorname{PSL}(2, R)$, where $R$ is a finitely generated ring in a number field $k$. Let $H$ and $K$ be abelian subgroups of $\Gamma$, and let $g \in \Gamma$. The double coset $H g K$ is closed in the congruence topology on $\Gamma$, with respect to $\rho$ and $R$, if one of the following conditions is satisfied.

- The groups $H$ and $K$ are both loxodromic subgroups of $\Gamma$.

- Exactly one of $\{H, K\}$ is a loxodromic subgroup of $\Gamma$ and the other is a maximal parabolic subgroup of $\Gamma$.

- Exactly one of $\{H, K\}$ is a loxodromic subgroup of $\Gamma$ and the other is a maximal cyclic parabolic subgroup of $\Gamma$.

Remark 3.6. In Corollary 3.5, we do not require any of the loxodromic subgroups to be maximal.

Proof. Since $M=\mathbb{H}^{3} / \Gamma$ is a Kleinian group of finite covolume, there exists a discrete, faithful representation from $\Gamma$ into $\operatorname{PSL}(2, \mathbb{C})$ such that the coefficient field of the image of $\Gamma$ is a finite field extension of $\mathbb{Q}$. By fixing one such representation $\rho$ we may view $\Gamma \subset \operatorname{PSL}(2, R) \subset \operatorname{PSL}(2, k)$, where $R$ is a finitely 
generated ring in a number field $k$. In the proof of Theorem 3.2, given an abelian subgroup $H$ of $\Gamma$, we adjoin finitely many algebraic numbers to $R$, if necessary, and then conjugate $\Gamma$ in $\operatorname{PSL}(2, R)$ such that $H$ has a nice form. We need to justify that we can replace our original representation $\rho$ with the new representation given by conjugation. (It is fine to replace our ring $R$ with a larger ring since any ring homomorphism from the larger ring restricts to a ring homomorphism from $R$.) To see this let $\alpha \in \operatorname{PSL}(2, R)$ and consider $\Gamma^{\prime}=\alpha \Gamma \alpha^{-1} \subset \operatorname{PSL}(2, R)$. Given a subset $X \subset \Gamma$ and an element $\gamma \in \Gamma-X$, let $X^{\prime}=\alpha X \alpha^{-1} \subset \Gamma^{\prime}$ and $\gamma^{\prime}=\alpha \gamma \alpha^{-1} \in \Gamma^{\prime}-X^{\prime}$. Suppose there exist a finite ring $S$ and a ring homomorphism $R \rightarrow S$, such that, under the induced group homomorphism

$$
\eta: \operatorname{PSL}(2, R) \rightarrow \operatorname{PSL}(2, S)
$$

$\eta\left(\gamma^{\prime}\right) \notin \eta\left(X^{\prime}\right)$. Then restricting $\eta$ to $\Gamma$ gives a congruence homomorphism

$$
\Gamma \hookrightarrow \operatorname{PSL}(2, R) \rightarrow \operatorname{PSL}(2, S)
$$

such that $\eta(\gamma) \notin \eta(X)$. We conclude that, for the purposes of our proof, it is legitimate to adjoin finitely many algebraic numbers to $R$ and then replace $\Gamma$ with a conjugate of $\Gamma$ in $\operatorname{PSL}(2, R)$.

We first show that loxodromic subgroups, maximal parabolic subgroups, and maximal cyclic parabolic subgroups are closed in the congruence topology on $\Gamma$, with respect to $\rho$ and $R$. This is well-known and the proof of some of the cases is in 3 . We include the proof here for the convenience of the reader.

Let $A$ be a maximal abelian subgroup of $\Gamma$. As above, we view $\Gamma \subset$ $\operatorname{PSL}(2, R) \subset \operatorname{PSL}(2, k)$, where $R$ is a finitely generated ring in a number field $k$. After adjoining elements to $R$ and $k$, if necessary, we may conjugate $\Gamma$ in $\operatorname{PSL}(2, R)$ such that $A$ is upper triangular. Let

$$
\gamma= \pm\left(\begin{array}{ll}
a & b \\
c & d
\end{array}\right) \in \Gamma-A
$$

be given. Since $\Gamma$ is discrete and $A$ is a maximal abelian subgroup of $\Gamma, \gamma$ and $A$ do not share a fixed point on the sphere at infinity. Therefore, $c \neq 0$. Let $I$ be an ideal of $R$ that does not divide $c$ and consider the congruence map

$$
\eta: \Gamma \hookrightarrow \operatorname{PSL}(2, R) \rightarrow \operatorname{PSL}(2, R / I)
$$

Then $\eta(\gamma) \notin \eta(A)$.

Let $H$ be a loxodromic subgroup of $\Gamma$. Let $A$ be the maximal abelian subgroup of $\Gamma$ containing $H$, and fix $\gamma \in \Gamma-H$. By the case above, we may assume that $\gamma \in A-H$. Write $H=\left\langle h^{m}\right\rangle$, where $h$ is a loxodromic element that generates $A$ and $m$ is a positive integer. Since $\gamma \in A-H, \gamma=h^{a}$, for some integer $a$ that is not divisible by $m$. By Corollary 2.3, there exists a congruence map

$$
\eta: \Gamma \hookrightarrow \operatorname{PSL}(2, R) \rightarrow \operatorname{PSL}(2, R / I)
$$

such that the order of $\eta(h)$ is divisible by $m$. Then $\eta(\gamma) \notin \eta(H)$. 
Let $H$ be a maximal cyclic parabolic subgroup of $\Gamma$. Choose $k \in \Gamma$ such that $A=\langle h, k\rangle$ is the maximal abelian subgroup of $\Gamma$ containing $H$, and fix $\gamma \in \Gamma-H$. By the case above, we may assume that $\gamma \in A-H$. Write $\gamma=h^{a} k^{b}$, $a, b \in \mathbb{Z}$. Since $\gamma \notin H, b \neq 0$. By Case 4 of Theorem 3.2 , for infinitely many primes $p$, there exist congruence maps

$$
\eta_{p}: \Gamma \hookrightarrow \operatorname{PSL}(2, R) \rightarrow \operatorname{PSL}\left(2, \mathbb{F}_{p}\right)
$$

such that $\eta_{p}(A) \cong \mathbb{Z} / p \mathbb{Z} \oplus \mathbb{Z} / p \mathbb{Z}$. If $p$ does not divide $b$, then $\eta_{p}(\gamma) \notin \eta_{p}(H)$.

Let $H, K$ and $g$ be given as in the statement of the corollary. Since the congruence topology on $\Gamma$ is equivariant under left and right multiplication, it suffices to show that the double coset $H K$ is closed in the congruence topology. By the argument above, we may assume that $H$ and $K$ do not commute. If $H$ and $K$ are both loxodromic, then the argument follows from Case 1 of Theorem 3.2. If $H$ is loxodromic and $K$ is a maximal parabolic subgroup of $\Gamma$, then the argument follows from the proof of Lemma 3.2 of [15. If $H$ is loxodromic and $K$ is a maximal cyclic parabolic subgroup of $\Gamma$, then the argument follows from Case 4 of Theorem 3.2

\section{Proof of conjugacy separability}

For a group $G$, we denote by $\widehat{G}$ the profinite completion of $G$. For a subgroup $H$ of $G$, we denote by $\bar{H}$ the closure of $H$ in $\widehat{G}$. The proof of Theorem 1.2 relies on the main technical theorem of [32, which we state here for convenience. We refer the reader to 32 for the definitions of the terms efficient and profinitely acylindrical used in it.

Theorem 4.1 ([32, Theorem 5.2). Let $\mathcal{G}$ be a finite graph of groups with conjugacy separable vertex groups. Let $G=\pi_{1}(\mathcal{G})$, and suppose that the profinite topology on $G$ is efficient and that $\mathcal{G}$ is profinitely 2-acylindrical. For any vertex $v$ of $\mathcal{G}$ and incident edges $e$ and $f$, suppose furthermore that the following conditions hold:

1. for any $g \in G_{v}$ the double coset $G_{e} g G_{f}$ is separable in $G_{v}$;

2. the edge group $G_{e}$ is conjugacy distinguished in $G_{v}$;

3. the intersection of the closures of $G_{e}$ and $G_{f}$ in the profinite completion of $G_{v}$ is equal to the profinite completion of their intersection. That is, the natural map ${\widehat{G_{e} \cap G_{f}}}_{\rightarrow} \bar{G}_{e} \cap \bar{G}_{f}$ is an isomorphism.

Then $G$ is conjugacy separable.

Definition 4.2. A subgroup $H \subseteq G$ is called conjugacy distinguished if, whenever $g \in G$ is not conjugate into $H$, there is a finite quotient of $G$ in which the image of $g$ is not conjugate into the image of $H$. 
As in [32, we will apply Theorem 4.1 to the torus decomposition of $M$. Henceforth, $M$ denotes a closed, orientable, Haken 3-manifold. Let $G=\pi_{1}(M)$ and let $\mathcal{G}$ be the graph-of-groups decomposition of $G$ induced by the torus decomposition of $M$.

Theorem 4.3 ([32, Theorem A). For $M$ as above, the profinite topology of the fundamental group of the graph of groups $\mathcal{G}$ is efficient.

It is convenient to make the extra assumption that every Seifert-fibered piece of the torus decomposition of $M$ is large - that is, has a fundamental group that virtually surjects a non-abelian free group. In this case, it turns out that $\mathcal{G}$ is profinitely 2-acylindrical, and so Theorem 4.1 applies. In 32, the remaining hypotheses of Theorem 4.1 were checked for graph manifolds. We will prove the corresponding results about the fundamental groups of hyperbolic manifolds.

Therefore, we need to consider $G_{v}=\Gamma$, the fundamental group of a finitevolume hyperbolic 3-manifold $N$. The incident edge groups $G_{e}$ and $G_{v}$ are maximal parabolic subgroups of $\Gamma$, which we shall denote $P$ and $Q$. The next lemma is a consequence of Theorem 1.4 .

Lemma 4.4. For any $g \in \Gamma$, the double coset $P g Q$ is separable in $\Gamma$.

Lemma 4.5. The subgroup $P$ is conjugacy distinguished in $\Gamma$.

Proof. As in the proof of Theorem 3.2, we may view $\Gamma \subset \mathrm{SL}(2, R) \subset \mathrm{SL}(2, k)$, where $R$ is a finitely generated ring contained in a number field $k$. Since $R$ is finitely generated, $R \subset \mathcal{O}_{\mathfrak{k}_{\mathfrak{p}}}$ for all but finitely many primes $\mathfrak{p}$ of $\mathcal{O}_{\mathfrak{k}_{\mathfrak{p}}}$. For each of these primes $\mathfrak{p}$ the residue class field map $\eta_{\mathfrak{p}}: \mathcal{O}_{\mathfrak{k}_{\mathfrak{p}}} \rightarrow F_{\mathfrak{p}}$ induces a group homomorphism

$$
\bar{\eta}_{\mathfrak{p}}: \Gamma \hookrightarrow \mathrm{SL}\left(2, \mathcal{O}_{\mathrm{k}_{\mathfrak{p}}}\right) \rightarrow \mathrm{SL}\left(2, F_{\mathfrak{p}}\right),
$$

where $F_{\mathfrak{p}}$ is the residue class field of $\mathcal{O}_{\mathrm{k}_{\mathfrak{p}}}$.

Let $\gamma$ be an element of $\Gamma$ that is not conjugate into $P$. If $\gamma$ is loxodromic, then the square of the trace of $\gamma, \operatorname{tr}(\gamma)^{2}$, is not equal to 4 . Choose a prime $\mathfrak{p}$ of $\mathcal{O}_{\mathrm{k}_{\mathfrak{p}}}$ such that $R \subset \mathcal{O}_{\mathrm{k}_{\mathfrak{p}}}$ and $\eta_{\mathfrak{p}}\left(\operatorname{tr}(\gamma)^{2}\right) \neq 4$. Then $\bar{\eta}_{\mathfrak{p}}(\gamma) \notin \bar{\eta}_{\mathfrak{p}}(P)$, as required. Now suppose that $\gamma$ is parabolic. As discussed in Case 3 of Theorem 3.2, we may view $N$ as the interior of a compact manifold $N^{\prime}$ with a finite number of tori boundary components. There is a one to one correspondence between the boundary components of $N^{\prime}$ and the conjugacy classes of maximal parabolic subgroups of $\Gamma$. Let $T_{\gamma}$ be the boundary component corresponding to $\gamma$ and let $T_{P}$ be the boundary component corresponding to $P$. Since $\gamma$ is not conjugate into $P$ and $P$ is a maximal parabolic subgroup, $T_{\gamma} \neq T_{P}$. By Thurston's Hyperbolic Dehn Surgery Theorem, there exists a complete hyperbolic manifold $M$ of finite volume obtained by Dehn surgery on $N^{\prime}$ along $T_{\gamma}$. We may choose $M$ such that the image of $\gamma$ is non-trivial in $\pi_{1}(M)$. Let

$$
\phi: \Gamma \cong \pi_{1}\left(N^{\prime}\right) \rightarrow \pi_{1}(M)
$$

be the homomorphism induced by inclusion $N^{\prime} \rightarrow M$. Then $\phi(P)$ is a maximal parabolic subgroup of $\pi_{1}(M)$ and $\phi(\gamma)$ is loxodromic. The argument then follows as above. 
Lemma 4.6. Let $P$ and $Q$ be non-conjugate maximal parabolic subgroups of $\Gamma$. There exists a sequence of group homomorphisms $f_{n}$ from $\Gamma$ to finite groups with the following properties.

1. For any finite index subgroup $K$ of $P$, there exists an $n$ with $\operatorname{Ker}\left(f_{n}\right) \cap P \subset$ $K$.

2. For every $n$, the intersection of $f_{n}(P)$ and $f_{n}(Q)$ is trivial.

Proof. As in the proof of Lemma 4.5 we may view $N$ as the interior of a compact manifold $N^{\prime}$ with a finite number of tori boundary components, each corresponding to a conjugacy class of a maximal parabolic subgroup of $\Gamma$. Let $T_{P}$ and $T_{Q}$ denote the boundary components of $N^{\prime}$ corresponding to $P$ and $Q$, respectively. By assumption, $T_{P} \neq T_{Q}$. Choose a basis $\left\{p_{1}, p_{2}\right\}$ of $P$ such that $N_{p_{1}}$ and $N_{p_{2}}$ are complete hyperbolic 3-manifolds of finite volume, where $N_{p_{1}}$ and $N_{p_{2}}$ are the manifolds obtained by Dehn surgery on $N^{\prime}$ along $P$ sending $p_{1}$ and $p_{2}$, respectively, to a meridian of the attached solid torus. Let

$$
\phi: \Gamma \cong \pi_{1}\left(N^{\prime}\right) \rightarrow \pi_{1}\left(N_{p_{1}}\right)
$$

be the homomorphism induced by inclusion. Then $\phi(Q)$ is a maximal parabolic subgroup of $\pi_{1}\left(N_{p_{1}}\right)$, and $\phi(P)$ is a loxodromic subgroup of $\pi_{1}\left(N_{p_{1}}\right)$ generated by $\phi\left(p_{2}\right)$. We then proceed as in Case 3 of Theorem 3.2. We may view $\pi_{1}\left(N_{p_{1}}\right) \subset$ $\mathrm{SL}\left(2, R_{1}\right)$, where $R_{1}$ is a finitely generated ring in a number field. Moreover, we may assume that

$$
\begin{gathered}
\phi\left(p_{2}\right)=\left(\begin{array}{cc}
\lambda & 0 \\
0 & \lambda^{-1}
\end{array}\right) \text { and } \\
\phi(Q)=\left\{\left(\begin{array}{ll}
a & b \\
c & d
\end{array}\right)\left(\begin{array}{ll}
1 & z \\
0 & 1
\end{array}\right)\left(\begin{array}{ll}
a & b \\
c & d
\end{array}\right)^{-1} \mid z \in A=\{m+n \beta \mid m, n \in \mathbb{Z}\}\right\},
\end{gathered}
$$

for some

$$
\left(\begin{array}{ll}
a & b \\
c & d
\end{array}\right) \in \mathrm{SL}\left(2, R_{1}\right) \text {, and } \lambda, \beta \in R_{1} .
$$

Since $\pi_{1}\left(N_{p_{1}}\right)$ is discrete, $\phi(P)$ and $\phi(Q)$ do not share a fixed point. Therefore, $a$ and $c$ are non-zero. Fix a natural number $n$. By Corollary 2.3, there exist a finite field $F_{n}$ and a ring homomorphism $\eta_{n}: R_{1} \rightarrow F_{n}$ such that the multiplicative order of $\eta_{n}(\lambda)$ is divisible by $n, \eta_{n}(a) \neq 0$ and $\eta_{n}(c) \neq 0$. This ring homomorphism induces a group homomorphism

$$
\bar{\eta}_{n}: \pi_{1}\left(N_{p_{1}}\right) \hookrightarrow \mathrm{SL}\left(2, R_{1}\right) \rightarrow \mathrm{SL}\left(2, F_{n}\right)
$$

such that the order of $\bar{\eta}_{n}\left(\phi\left(p_{2}\right)\right)$ is divisible by $n$. Moreover, since $\eta_{n}(a) \neq 0$ and $\eta_{n}(c) \neq 0$, the intersection of $\eta_{n}(\phi(\mathrm{P}))$ and $\eta_{n}(\phi(Q))$ is trivial. Let

$$
g_{n}: \Gamma \cong \pi_{1}\left(N^{\prime}\right) \rightarrow \pi_{1}\left(N_{p_{1}}\right) \rightarrow \operatorname{SL}\left(2, F_{n}\right)
$$


denote the composition $\bar{\eta}_{n} \circ \phi$. Then the intersection of $g_{n}(P)$ and $g_{n}(Q)$ is trivial, $g_{n}\left(p_{1}\right)$ is trivial, and $g_{n}\left(p_{2}\right)$ has order divisible by $n$. By a similar argument, there exist a finite field $L_{n}$ and a group homomorphism

$$
h_{n}: \Gamma \cong \pi_{1}\left(N^{\prime}\right) \rightarrow \pi_{1}\left(N_{p_{2}}\right) \rightarrow \mathrm{SL}\left(2, L_{n}\right)
$$

such that the intersection of $h_{n}(P)$ and $h_{n}(Q)$ is trivial, $h_{n}\left(p_{2}\right)$ is trivial, and $h_{n}\left(p_{1}\right)$ has order divisible by $n$. Let $K_{n}=\operatorname{Ker}\left(g_{n}\right) \cap \operatorname{Ker}\left(h_{n}\right)$, and let

$$
f_{n}: \Gamma \rightarrow \Gamma / K_{n}
$$

denote the quotient map. Then the intersection of $f_{n}(P)$ and $f_{n}(Q)$ is trivial, and $\operatorname{Ker}\left(f_{n}\right) \cap P=K_{n} \cap P \subset\left\langle n p_{1}, n p_{2}\right\rangle=n P$. The collection $\left\{f_{n}\right\}$ satisfies the conditions above, since given a subgroup $K$ of finite index in $P$, there exists a natural number $n$, such that $n P \subset K$.

Lemma 4.7. Let $P, Q$ be distinct maximal parabolic subgroups of $\Gamma$. The intersection of the closures $\bar{P} \cap \bar{Q}$ is trivial in the profinite completion $\widehat{\Gamma}$.

Proof. We first consider the case in which $P$ and $Q$ are not conjugate in $\Gamma$. By the universal property of the profinite completion, the maps $f_{n}: \Gamma \rightarrow \Gamma / K_{n}$ in Lemma 4.6 extend to continuous homomorphisms $\hat{f}_{n}$ from $\widehat{\Gamma}$. Let $f=\left(\hat{f}_{n}\right)$ : $\widehat{\Gamma} \rightarrow \prod_{n} \Gamma / K_{n}$ be the continuous homomorphism to the Cartesian product of $\Gamma / K_{n}$. By Lemma 4.6 its restriction to $\bar{P}$ and $\bar{Q}$ is injective and the image of $\bar{P} \cap \bar{Q}$ in $\Gamma / K_{n}$ is trivial. Therefore $\bar{P} \cap \bar{Q}$ is trivial.

Suppose now that $P$ and $Q$ are conjugate in $\Gamma$. As in Case 3 of the proof of Theorem 3.2, there is a subgroup $\Gamma_{0}$ of finite index in $\Gamma$ such that $P_{0}=\Gamma_{0} \cap P$ and $Q_{0}=\Gamma_{0} \cap Q$ are not conjugate in $\Gamma_{0}$. By the non-conjugate case, we have that $\bar{P}_{0} \cap \bar{Q}_{0}=1$ in the profinite completion $\widehat{\Gamma}_{0}$. But $\widehat{\Gamma}_{0}$ is a subgroup of finite index in $\widehat{\Gamma}$, and so $\bar{P} \cap \bar{Q}$ is periodic in $\widehat{\Gamma}$. But $\bar{P} \cong \widehat{\mathbb{Z}^{2}}$, which is torsion-free, and so $\bar{P} \cap \bar{Q}=1$ as required.

These lemmas complement the corresponding results for Seifert-fibered manifolds, which we list below for convenience. As usual, by a peripheral subgroup of the fundamental group of a 3-manifold we mean a subgroup that (up to conjugacy) corresponds to a boundary component of $N$.

Lemma 4.8 (27]). Double cosets of peripheral subgroups in Seifert-fibered 3manifold groups are separable.

Lemma 4.9 ([32], Lemma 5.3). Every peripheral subgroup of the fundamental group of a Seifert-fibered 3-manifold group is conjugacy distinguished.

Lemma 4.10 (32, Lemma 5.4). Let $N$ be a large Seifert-fibered 3-manifold and let $P, Q$ be distinct peripheral subgroups of $\pi_{1}(N)$. Then $\widehat{P} \cap \widehat{Q}=\widehat{Z}$, the profinite completion of the canonical normal cyclic subgroup of $\pi_{1}(N)$.

Proceeding exactly as in 32 , Lemmas 4.7 and 4.10 can be used together to generalize Lemma 5.5 of [32] as follows. 
Lemma 4.11. Let $M$ be a closed, orientable, Haken 3-manifold in which every Seifert-fibered piece of the torus decomposition is large. Let $G=\pi_{1}(M)$ and let $\mathcal{G}$ be the graph of groups induced by the torus decomposition of $M$. Then $\mathcal{G}$ is profinitely 2-acylindrical.

When every Seifert-fibered piece of $M$ is large, Theorem 1.2 is a direct consequence of Theorem 4.1, together with Theorem 4.3 and Lemmas 4.4. 4.5. 4.7, 4.8, 4.9, 4.10 and 4.11. Of course, we also need the Geometrization Theorem (proved in the Haken case by Thurston and in full by Perelman), which implies that every piece of the torus decomposition is either Seifert-fibered or hyperbolic.

Finally, proceeding exactly as in the proof of Theorem D of [32], the full statement of Theorem 1.2 follows.

\section{References}

[1] Ian Agol, Daniel Groves and Jason Manning, 'Residual finiteness, QCERF and fillings of hyperbolic groups', Geom. Topol. 13 (2009) $1043-1073$.

[2] Ian Agol, 'The Virtual Haken Conjecture', preprint (2012).

[3] E.S. Allman and E. Hamilton, 'Abelian subgroups of finitely generated Kleinian groups are separable', Bull. London Math. Soc. 31 (1999) 163 172 .

[4] R.C. Alperin, 'An elementary account of Selberg's Lemma', L'Enseignement Mathematique t.33 (1987) $269-273$.

[5] H. Bass, 'Groups of integral representation type', Pacific Journal of Math. 86, No.1 (1980) 15 - 50.

[6] R. Benedetti and C. Petronio, 'Lectures on Hyperbolic Geometry', (Springer-Verlag, 1992).

[7] Nicolas Bergeron. Virtual fibering of certain cover of $\mathbb{S}^{3}$, branched over the figure eight knot. C. R. Math. Acad. Sci. Paris, 346 (2008) 1073-1078.

[8] Nicolas Bergeron, Frédéric Haglund, and Daniel T. Wise. Hyperplane sections in arithmetic hyperbolic manifolds. Jour. London Math. Soc., 83 (2011) 431-448.

[9] R. G. Burns, A. Karrass, and D. Solitar. A note on groups with separable finitely generated subgroups. Bulletin of the Australian Mathematical Society, 36 (1987) 153-160.

[10] S. C Chagas and P. A Zalesskii. Bianchi groups are conjugacy separable. J. Pure and Applied Algebra, 214 (2010) 1696-1700.

[11] Eric Chesebro, Jason DeBlois, and Henry Wilton. Some virtually special hyperbolic 3-manifold groups. ArXiv:0903.5288, 2009. 
[12] M. Culler and P. Shalen, 'Varieties of group representations and splittings of 3-manifolds' Ann. of Math. 117 (1983) $109-146$.

[13] Frédéric Haglund and Daniel T. Wise. Special cube complexes. Geometric and Functional Analysis, 17 (2008) 1551-1620.

[14] E. Hamilton, 'Abelian subgroup separability of Haken 3-manifolds and closed hyperbolic $n$-orbifolds', Proc. London Math. Soc. (3) 83 (2001) 626646.

[15] E. Hamilton, 'Classes of separable two-generator free subgroups of 3manifold groups', Topology Appl., 131 (2003) 239 - 254.

[16] E. Hamilton, 'Finite quotients of rings and applications to subgroup separability of linear groups', Trans. Amer. Math. Soc. (5) 357 (2004) 1995 2006.

[17] J. Hempel, 'Residual finiteness for 3-manifolds', Combinatorial group theory and topology, Gersten, S.M., Stallings, J.R., (editors), Ann. Math. Stud. No. 111 (1987) 379-396.

[18] William H. Jaco and Peter B. Shalen. Seifert fibered spaces in 3-manifolds. Memoirs of the American Mathematical Society, 21(220):viii+192, 1979.

[19] G.J. Janusz, 'Algebraic Number Fields', (Academic Press, 1973).

[20] Klaus Johannson. Homotopy equivalences of 3-manifolds with boundaries, volume 761 of Lecture Notes in Mathematics. Springer, Berlin, 1979.

[21] J. Kahn and V. Markovic, 'Immersing almost geodesic surfaces in a closed hyperbolic 3-manifold', Annals of Mathematics, to appear.

[22] D.D. Long and G.A. Niblo, 'Subgroup separability and 3-manifold groups', Math. Z. 207 (1991) $209-215$.

[23] A.I. Mal'cev, 'On homomorphisms to finite groups' American Mathematical Society Translations, Series 2, 119 (1983) $67-79$.

[24] Armando Martino. A proof that all Seifert 3-manifold groups and all virtual surface groups are conjugacy separable. J. Algebra, 313 (2007) 773-781.

[25] Ashot Minasyan. Separable subsets of GFERF negatively curved groups. J. Algebra, 304 (2006) 1090-1100.

[26] Ashot Minasyan. Hereditary conjugacy separability of right angled Artin groups and its applications. Groups geometry and Dynamics (to appear). ArXiv:0905.1282, May 2009.

[27] G.A. Niblo, 'Separability properties of free groups and surface groups', Journal of Pure and Applied Algebra 78 (1992) 77 - 84. 
[28] V. N. Remeslennikov. Conjugacy in polycyclic groups. Akademiya Nauk SSSR. Sibirskoe Otdelenie. Institut Matematiki. Algebra i Logika, 8 (1969) $712-725$.

[29] P. Scott, 'Subgroups of surface groups are almost geometric', J. London Math. Soc. 17 (1978) 555-565.

[30] P. Shalen, 'Orders of elements in finite quotients of Kleinian groups', preprint.

[31] P. F. Stebe. A residual property of certain groups. Proceedings of the American Mathematical Society, 26:37-42, 1970.

[32] Henry Wilton and Pavel Zalesskii. Profinite properties of graph manifolds. Geometriae Dedicata, 147 (2010) 29-45.

[33] D.T. Wise. Research announcement: the structure of groups with a quasiconvex hierarchy. Electron. Res. Announc. Math. Sci., 16 (2009) 44-55.

[34] Daniel T. Wise. 'The structure of groups with a quasiconvex hierarchy', preprint, http://comet. lehman. cuny. edu/behrstock/cbms/program.html.

Department of Mathematics and Computer Science

Emory University

Atlanta, GA 30322

USA

Department of Mathematics

University College London

Gower Street

London

WC1E 6BT

UK

Department of Mathematics

University of Brasilia

70910-900 Brasilia-DF

Brazil 